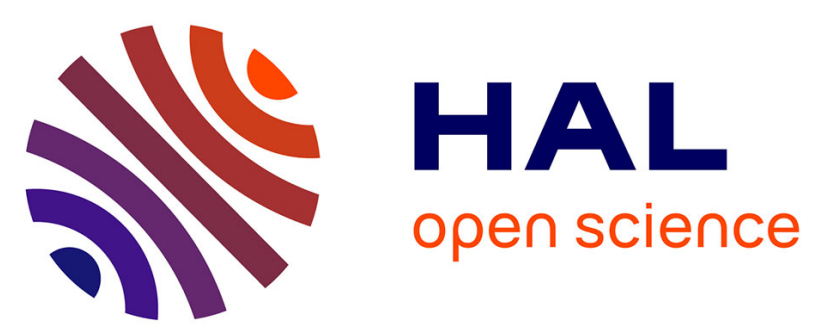

\title{
Temperature dependences of mechanisms responsible for the water-vapor continuum absorption. II. Dimers and collision-induced absorption
}

\author{
Claude Leforestier, Richard Tipping, Q. Ma
}

\section{- To cite this version:}

Claude Leforestier, Richard Tipping, Q. Ma. Temperature dependences of mechanisms responsible for the water-vapor continuum absorption. II. Dimers and collision-induced absorption. Journal of Chemical Physics, 2010, 132 (16), pp.164302. 10.1063/1.3384653 . hal-00490187

\section{HAL Id: hal-00490187 https://hal.science/hal-00490187}

Submitted on 9 Jun 2021

HAL is a multi-disciplinary open access archive for the deposit and dissemination of scientific research documents, whether they are published or not. The documents may come from teaching and research institutions in France or abroad, or from public or private research centers.
L'archive ouverte pluridisciplinaire HAL, est destinée au dépôt et à la diffusion de documents scientifiques de niveau recherche, publiés ou non, émanant des établissements d'enseignement et de recherche français ou étrangers, des laboratoires publics ou privés. 
See discussions, stats, and author profiles for this publication at: https://www.researchgate.net/publication/44572049

\section{Temperature dependences of mechanisms responsible for the water-vapor continuum absorption. II. Dimers and collision-induced absorption}

Article in The Journal of Chemical Physics · April 2010

DOI: $10.1063 / 1.3384653$ · Source: PubMed

CITATIONS

31

3 authors:

Claude Leforestier

Université de Montpellier

120 PUBLICATIONS 6,164 CITATIONS

SEE PROFILE

Quanlin Ma

99 PUBLICATIONS 2,037 CITATIONS

SEE PROFILE
READS

46

Rh Tipping

University of Alabama

130 PUBLICATIONS 4,202 CITATIONS

SEE PROFILE 


\title{
Temperature dependences of mechanisms responsible for the water-vapor continuum absorption. II. Dimers and collision-induced absorption
}

\author{
C. Leforestier, ${ }^{1}$ R. H. Tipping, ${ }^{2}$ and Q. Ma ${ }^{3, a)}$ \\ ${ }^{1}$ Institut Charles Gerhardt CNRS-5253, CC15.01, Université Montpellier 2, 34095 Montpellier, France \\ ${ }^{2}$ Department of Physics and Astronomy, University of Alabama, Tuscaloosa, Alabama 35487-0324, USA \\ ${ }^{3}$ NASA/Goddard Institute for Space Studies and Department of Applied Physics and Applied \\ Mathematics, Columbia University, 2880 Broadway, New York, New York 10025, USA
}

(Received 28 January 2010; accepted 18 March 2010; published online 22 April 2010)

\begin{abstract}
We investigated the magnitude and temperature dependence ( $T$ dependence) of the dimer absorption in the region of $0-600 \mathrm{~cm}^{-1}$ and the collision-induced absorption (CIA) in the region of $0-1150 \mathrm{~cm}^{-1}$. Together with our previous study of the self water-vapor continuum contributions resulting from far-wing line shapes of the allowed $\mathrm{H}_{2} \mathrm{O}$ lines in the infrared window between 800 and $1150 \mathrm{~cm}^{-1}$, we find that the three mechanisms have completely different $T$ dependence behaviors. The dimer absorption has the strongest negative $T$ dependence and the continuum absorption from far wings of the allowed lines has a moderately strong negative one. Meanwhile, the CIA exhibits a mild $T$ dependence. In addition, their $T$ dependence patterns are quite different. The $T$ dependence of the far-wing theory varies significantly as the frequency of interest $\omega$ varies. For CIA, in general, its $T$ dependence is mildly negative, but becomes slightly positive in the window region between the $\mathrm{H}_{2} \mathrm{O}$ bands. In contrast, the $T$ dependence of the dimer absorption varies slightly as $\omega$ varies. In the microwave and submillimeter region, its $T$ dependence becomes uniform. Concerning the relative importance for each of these three mechanisms, we find that in the infrared widow, the far-wing contributions are the dominant source of the self-continuum. Within the band, its contributions are definitely responsible for the measured continuum data. But, it is impossible to draw quantitatively conclusions on its relative importance unless one is able to improve the accuracy of the local line calculations significantly. On the other hand, within the pure rotational band, the dimer absorptions are a minor contributor to the self-continuum measurements, and its role becomes more important in the microwave and submillimeter regions. Finally, based on our study we conclude that contributions to the self-continuum from CIA in the frequency region of 0-1150 $\mathrm{cm}^{-1}$ are negligible. (C) 2010 American Institute of Physics. [doi:10.1063/1.3384653]
\end{abstract}

\section{INTRODUCTION}

In a previous paper, ${ }^{1}$ we presented new theoretical calculations for the far-wing absorption for $\mathrm{H}_{2} \mathrm{O}-\mathrm{H}_{2} \mathrm{O}$ pairs and applied these to study the temperature dependence ( $T$ dependence) of the water-vapor continuum. We compared our results with the experimental data available in the $800-1150 \mathrm{~cm}^{-1}$ spectral region and found good agreement, both magnitude and $T$ dependence, with the most recent results by Barynov et al. for temperatures ranging from 310.8 to $363.6 \mathrm{~K}^{2}$ Because the continuum scales with the number density squared and the saturated vapor pressure decreases rapidly with $T$, it is harder to make accurate experimental measurements as $T$ decreases. However, for applications in Earth's atmosphere, one needs accurate results down to approximately $200 \mathrm{~K}$. Therefore, it is important to know the $T$ dependence of the water-vapor continuum because it can serve as a guide in predicting absorptions at lower temperatures based on data obtained at room temperature and above.

When the water-vapor continuum was first discovered by Elsasser, ${ }^{3}$ it was speculated that the physical mechanism re-

\footnotetext{
${ }^{a)}$ Electronic mail: qma@giss.nasa.gov.
}

sponsible was the superposition of the far wings of the allowed monomer lines. However, since that time, two other physical mechanisms have been proposed as contributors, minor or even major, to the water-vapor continuum: water dimers and collision-induced absorption (CIA).

The first is dimer absorption, that has been postulated as a major contributor to the self-continuum in the $8-12 \mu \mathrm{m}$ atmospheric window region. ${ }^{4,5}$ This was bolstered by a specious argument based on the "characteristic temperature" $T_{0} \approx 1800 \mathrm{~K}$ found by Roberts $\mathrm{et} \mathrm{al.}^{6}$ for the self-continuum and corresponds approximately to the hydrogen bond energy between two water monomers. This was discussed at length in our previous paper and will not be repeated here. Recently, Scribano et al. ${ }^{7,8}$ have calculated more accurate values for the number density of water dimers and the corresponding absorption in the $0-945 \mathrm{~cm}^{-1}$ spectral region. They found that between 20 and $500 \mathrm{~cm}^{-1}$ the dimer contribution to the water-vapor continuum is approximately $15 \%-20 \%$, but falls off very quickly above $600 \mathrm{~cm}^{-1}$ and is negligible in the microwindow around $944 \mathrm{~cm}^{-1}$. Like the far-wing mechanisms discussed previously, ${ }^{1}$ the dimer absorption scales as the square of the monomer number density, but will have a much different $T$ dependence. 
The second is CIA due to transient dipole moments induced during binary (or higher-order) collisions. Because the lifetime of these dipoles is short (duration of a collision), the corresponding spectral lines are very broad, and except for very light molecular pairs (e.g., $\left.\mathrm{H}_{2}-\mathrm{H}_{2}\right)$, the widths are larger than the separations between lines; thus, the corresponding spectra appear as smooth envelopes. It is well known that $\mathrm{N}_{2}-\mathrm{N}_{2}, \mathrm{O}_{2}-\mathrm{O}_{2}$, and $\mathrm{N}_{2}-\mathrm{O}_{2}$ pairs whose spectra have been measured in the laboratory and calculated theoretically play a role in atmospheric spectra. ${ }^{9-11}$ The CIA for $\mathrm{H}_{2} \mathrm{O}-\mathrm{N}_{2}$ has been calculated theoretically in the $0-2000 \mathrm{~cm}^{-1}$ spectral range. ${ }^{12}$ It was shown that the contribution of this mechanism was an order of magnitude or more less than the observed foreign continuum. Also, because the polarizability tensor for $\mathrm{O}_{2}$ is comparable to that of $\mathrm{N}_{2}$ while the number density of $\mathrm{O}_{2}$ is approximately $1 / 4$ that of $\mathrm{N}_{2}$ in the atmosphere, the $\mathrm{H}_{2} \mathrm{O}-\mathrm{O}_{2}$ continuum will also be negligible. It is worth noting here that in collision-induced spectra, it is possible for each molecule to make a rotational or vibration-rotational transition (double transitions) that appears at the sum of the individual wavenumbers; ${ }^{13}$ this could shift the absorption into a spectral region where other atmospheric absorptions are small, and thus could play a role. Such double transitions were considered for $\mathrm{H}_{2} \mathrm{O}-\mathrm{N}_{2}$, in which the water molecule makes a rotational transition, while the $\mathrm{N}_{2}$ make a fundamental vibration-rotational transition. This has CIA where the high-frequency wing of the $4.3 \mu \mathrm{m}$ band of $\mathrm{CO}_{2}$ falls off very rapidly and the highfrequency wing of $\mathrm{N}_{2}-\mathrm{N}_{2}$ has been observed in atmospheric measurements. ${ }^{14}$ Thus, the observed spectra should exhibit humidity dependence, although their magnitude would be small.

In this paper, we present the first calculations of the CIA spectrum of $\mathrm{H}_{2} \mathrm{O}-\mathrm{H}_{2} \mathrm{O}$ and its $T$ dependence. Like the other mechanisms mentioned above, CIA also depends on the number density squared, however, its $T$ dependence will be different, and this could provide a method to distinguish it from the other mechanisms.

The present paper is organized as follows. In Sec. II we present new results for the dimer absorption. In Sec. III, we discuss the CIA spectrum of $\mathrm{H}_{2} \mathrm{O}-\mathrm{H}_{2} \mathrm{O}$. Finally, in Sec. IV, we present a short discussion of the results obtained and conclusions from this work.

\section{WATER DIMER ABSORPTION}

Based on the dimer theory developed previously, ${ }^{8}$ the dimer absorption coefficient $\alpha(\omega)$ can be expressed as

$$
\begin{aligned}
\alpha(\omega)= & \frac{4 \pi^{2}}{3 \hbar c} n_{D} \sum_{n^{\prime \prime}} \sum_{n^{\prime}>n^{\prime \prime}} \omega_{n^{\prime} n^{\prime \prime}} \frac{g_{n^{\prime \prime}}}{Q_{\mathrm{RV}}^{D}}\left(e^{-E_{n^{\prime \prime}} / k_{B} T}-e^{-E_{n^{\prime}} / k_{B} T}\right) \\
& \times S_{n^{\prime \prime} n^{\prime}} \mathcal{L}\left(\omega, \omega_{n^{\prime} n^{\prime \prime}}, \gamma\right),
\end{aligned}
$$

where $n_{D}$ is the number density of the dimers, $n^{\prime \prime}$ and $n^{\prime}$ are simple notations for a set of quantum numbers required to specify states of the dimer, $\omega_{n^{\prime} n^{\prime \prime}}\left(=E_{n^{\prime}}-E_{n^{\prime \prime}}>0\right)$ are the resonant frequencies (in $\mathrm{cm}^{-1}$ ) for transitions $n^{\prime \prime} \rightarrow n^{\prime}, g_{n^{\prime \prime}}$ is the normalized space and nuclear spin degeneracy factor of state $n^{\prime \prime}$, and $Q_{\mathrm{RV}}^{D}$ is the rotation-vibration partition function of the dimer. In the above expression, $S_{n^{\prime \prime} n^{\prime}}$ are the dimer line strength factors and $\mathcal{L}\left(\omega, \omega_{n^{\prime} n^{\prime \prime}}, \gamma\right)$ is the normalized line shape function. It has been known for years that determining the number density of the dimer at the temperatures of interest is a crucial problem in developing the dimer theory. In terms of the equilibrium constant $K_{P}(T)$ (in units of atm ${ }^{-1}$ ) defined by

$$
K_{P}(T) \equiv \frac{P_{D}}{P_{M}^{2}},
$$

the number density of the dimers is given by

$$
n_{D}=k_{B} T n_{M}^{2} K_{P}(T)=n_{M} P_{M} K_{P}(T)=\frac{P_{M}^{2}}{k_{B} T} K_{P}(T) .
$$

In the above expressions, $P_{D}$ and $P_{M}\left(=P_{\mathrm{H} 2 \mathrm{O}}\right)$ are the pressures of dimers and monomers respectively, and $n_{M}$ is the number density of the $\mathrm{H}_{2} \mathrm{O}$ monomer. Recently, progress on how $K_{P}(T)$ varies with the temperature has been made. ${ }^{7}$ It is this achievement that allows us to go a step further in studying the $T$ dependence of the absorption coefficient.

\section{A. Dominant player $K_{P}(T) / Q_{\mathrm{RV}}^{D}$ in determining the $T$ dependence of the dimer absorption}

According to the paper, ${ }^{7}$ one is able to derive the canonical expression for $K_{P}(T)$ as

$$
K_{P}(T)=\frac{1}{k_{B} T} \cdot \frac{Q_{\mathrm{RV}}^{D} e^{D_{0} / k_{B} T}}{\lambda_{D}^{3}} \cdot\left(\frac{\lambda_{M}^{3}}{Q_{\mathrm{RV}}^{M}}\right)^{2},
$$

where $\lambda_{X}=h / \sqrt{2 \pi m_{X} k_{B} T}$ is the thermal de Broglie wavelength. The exponential term $\exp \left(D_{0} / k_{B} T\right)$ accounts for the difference in the dimer's $\left(V_{0}=-D_{0}\right)$ and monomer's $\left(V_{0}=0\right)$ zeros of vibrational energies. A $D_{0}$ value of $3.52 \mathrm{kcal} / \mathrm{mol}$ (i.e., $1771 \mathrm{~K}$ ) was obtained from rigorous quantum calculations using a semiempirical potential energy surface previously fitted to spectroscopic results. ${ }^{15}$ The most recent estimate, based on high accuracy ab initio calculations, leads to the somewhat reduced value of $3.13 \mathrm{kcal} / \mathrm{mol}$ (i.e., 1575 K). ${ }^{16}$ By contrast, a fitting value of $3.27 \mathrm{kcal} / \mathrm{mol}$ (i.e., 1646 $\mathrm{K})$ of $D_{0}$ was reported in the literature. ${ }^{17}$ By taking into account that

$$
\frac{\lambda_{M}^{6}}{\lambda_{D}^{3}}=\frac{h^{3}}{\left(\pi m_{M} k_{B} T\right)^{3 / 2}},
$$

one can write $K_{P}(T) / Q_{\mathrm{RV}}^{D}$ as

$$
\frac{K_{P}(T)}{Q_{\mathrm{RV}}^{D}}=\frac{h^{3} e^{D_{0} / k_{B} T}}{k_{B} T\left(\pi m_{M} k_{B} T\right)^{3 / 2}\left(Q_{\mathrm{RV}}^{M}\right)^{2}} .
$$

For temperatures of interest in atmospheric applications, the rotation-vibration partition function of the $\mathrm{H}_{2} \mathrm{O}$ monomer $Q_{\mathrm{RV}}^{M}$ can be replaced by the rotational partition function $Q_{R}^{M}$ which varies with $T$ as $T^{3 / 2}$. For later use, we give here its expression $^{18}$ 
TABLE I. Averaged values of the parameters $\mu_{\Delta K}^{\Gamma}, \alpha_{\Delta K}^{\Gamma}$, and $\beta_{\Delta K}^{\Gamma}$ in Eq. (12) and associated dispersions ( $\mu$ in Debye, $\alpha$ and $\beta$ in $\left.1 / \mathrm{cm}^{-1}\right)$. Averaging is performed over the lowest 150 vibrational eigenstates $\Phi_{\nu K}^{J \Gamma}(\nu$ $=0, \cdots, 149)$ with $J=0, \cdots, 5$ and $K=0, \cdots, J$ for all irreducible representations $\Gamma$.

\begin{tabular}{lcccccc}
\hline \hline & $\mu_{\Delta K=0}$ & $\alpha_{\Delta K=0}$ & $\beta_{\Delta K=0}$ & $\mu_{\Delta K=1}$ & $\alpha_{\Delta K=1}$ & $\beta_{\Delta K=1}$ \\
\hline Average & 1.250 & $2.43 \times 10^{-3}$ & $1.11 \times 10^{-2}$ & 0.196 & $1.83 \times 10^{-3}$ & $4.09 \times 10^{-3}$ \\
Dispersion & $1.04 \times 10^{-2}$ & $3.67 \times 10^{-4}$ & $8.57 \times 10^{-4}$ & $1.99 \times 10^{-2}$ & $2.61 \times 10^{-4}$ & $6.61 \times 10^{-4}$ \\
\hline \hline
\end{tabular}

$$
Q_{R}^{M}=\frac{\sqrt{\pi}}{2}\left(\frac{T^{3}}{\Theta_{A} \Theta_{B} \Theta_{C}}\right)^{1 / 2}
$$

where the $\Theta$ 's correspond to the monomer rotational constants. Then concerning the $T$ dependence, $K_{P}(T) / Q_{\mathrm{RV}}^{D}$ can be well described as

$$
\frac{K_{P}(T)}{Q_{\mathrm{RV}}^{D}}=G \cdot T^{-11 / 2} e^{D_{0} / k_{B} T},
$$

where $G$ is a constant. As shown by Eq. (8), the factor of $K_{P}(T) / Q_{\mathrm{RV}}^{D}$ exhibits a very strong negative $T$ dependence because it varies as $T^{-11 / 2} \times \exp \left(D_{0} / k_{B} T\right)$. It plays the dominant role for the $T$ dependence of the dimer absorption coefficient.

\section{B. The dimer number density $n_{D}$ without the factor $K_{P}(T)$}

Similar to what we have explained in Paper $\mathrm{I},{ }^{1}$ whether the remaining part of $n_{D}$ without the $K_{P}(T)$ factor would have any $T$ dependence or not depends on the units used for the absorption coefficient $\alpha(\omega)$. As shown by the second expressions for $n_{D}$ in Eq. (3), if $\mathrm{cm}^{2}$ molecule $\mathrm{atm}^{-1}$ is used as the units for $\alpha(\omega)$, the remaining part of $n_{D}$ would not contribute to the $T$ dependence. Meanwhile, if one chooses $\mathrm{dB} / \mathrm{km}$ as the unit of $\alpha(\omega)$, it would contribute a factor of $1 / T$ to the $T$ dependence of the absorption.

\section{A remaining factor $H(\omega, T)$}

By factoring out $K_{P}(T) / Q_{\mathrm{RV}}^{D}, n_{M} P_{M}$, and some constants from Eq. (1), the remaining part of $\alpha(\omega)$, which is denoted by $H(\omega, T)$, corresponds to summations over $n^{\prime \prime}$ and $n^{\prime}$ given by

$$
\begin{aligned}
H(\omega, T) \equiv & \sum_{n^{\prime \prime}} \sum_{n^{\prime}>n^{\prime \prime}} \omega_{n^{\prime} n^{\prime \prime}} g_{n^{\prime \prime}}\left(e^{-E_{n^{\prime \prime}} / k_{B} T}-e^{-E_{n^{\prime}} / k_{B} T}\right) \\
& \times S_{n^{\prime \prime} n^{\prime}} \mathcal{L}\left(\omega, \omega_{n^{\prime} n^{\prime \prime}}, \gamma\right) .
\end{aligned}
$$

It turns out that figuring out its $T$ dependence is the most difficult job in the present study. Two procedures are involved in this process. The first one is to simplify expressions for terms to be summed as much as possible. The second one is to reduce the summations over the two sets of quantum numbers $n^{\prime \prime}$ and $n^{\prime}$ into the two quantum energies $E_{n^{\prime \prime}}$ and $E_{n^{\prime}}$ and to transform the latter into continuous integrations.

Let us explain the first procedure. It has been shown ${ }^{8}$ that with the Van Vleck-Weisskopf line shape, varying the value of half-width $\gamma$ in the range of $0.12-4 \mathrm{~cm}^{-1}$ does not change the absorption spectrum appreciably. This insensitivity results from a huge number of dimer lines densely dis- tributed in the frequency region of interest. For example, at $T=298 \mathrm{~K}$, there are about $2 \times 10^{7}$ lines per $\mathrm{cm}^{-1}$ of the spectrum in the range $0-130 \mathrm{~cm}^{-1}$, and more than $10^{7}$ per $\mathrm{cm}^{-1}$ up to $380 \mathrm{~cm}^{-1}$. As a result, any normalized line shapes would yield similar spectra. For simplicity, this fact allows one to replace the line shape function $\mathcal{L}\left(\omega, \omega_{n^{\prime} n^{\prime \prime}}, \gamma\right)$ by the Dirac delta function,

$$
\begin{aligned}
& \lim _{\gamma \rightarrow 0} \frac{\omega}{\omega_{n^{\prime} n^{\prime \prime}}} \cdot \frac{1}{\pi}\left[\frac{\gamma}{\left(\omega-\omega_{n^{\prime} n^{\prime \prime}}\right)^{2}+\gamma^{2}}+\frac{\gamma}{\left(\omega+\omega_{n^{\prime} n^{\prime \prime}}\right)^{2}+\gamma^{2}}\right] \\
&=\delta\left(\omega-\omega_{n^{\prime} n^{\prime \prime}}\right)+\delta\left(\omega+\omega_{n^{\prime} n^{\prime \prime}}\right) .
\end{aligned}
$$

By doing this, we have completely ignored effects of the $T$ dependence of the absorption arising from the line shape.

The expression for the rotation-vibration line strength factor $S_{n^{\prime \prime} n^{\prime}}$ is given by

$$
S_{n^{\prime \prime} n^{\prime}}=\left\langle\Phi_{\nu^{\prime} K^{\prime}}^{J^{\prime} \Gamma^{\prime}}\left|\mu_{\Delta K}^{B F}\right| \Phi_{\nu^{\prime \prime} K^{\prime \prime}}^{J^{\prime \prime} \Gamma^{\prime \prime}}\right\rangle^{2} \times S\left(J^{\prime} K^{\prime} ; J^{\prime \prime} K^{\prime \prime}\right),
$$

where $\left|\Phi_{\nu K}^{J \Gamma}\right\rangle$ stands for the vibration wave function, $\Delta K$ $=K^{\prime}-K^{\prime \prime}$ and $S\left(J^{\prime} K^{\prime} ; J^{\prime \prime} K^{\prime \prime}\right)$ is the Hönl-London (HL) factor. It has been shown previously ${ }^{8}$ that at a fixed $\Delta K$ value, the dipole matrix elements $\left\langle\Phi_{\nu^{\prime} K^{\prime}}^{J^{\prime} \Gamma^{\prime}}\left|\mu_{\Delta K}^{B F}\right| \Phi_{\nu^{\prime \prime} K^{\prime \prime}}^{J^{\prime \prime} \Gamma^{\prime \prime}}\right\rangle$ are essentially independent of $J^{\prime \prime}$ and $J^{\prime}$, up to very high $J$ values and depend on $K^{\prime \prime}$ and $K^{\prime}$ only slightly (see Fig. 6 of Ref. 8). As a result, these matrix elements can be well reproduced by a simple interpolation function,

$$
\left\langle\Phi_{\nu^{\prime} K^{\prime}}^{J^{\prime} \Gamma^{\prime}}\left|\mu_{\Delta K}^{B F}\right| \Phi_{\nu^{\prime \prime} K^{\prime \prime}}^{J^{\prime \prime} \Gamma^{\prime \prime}}\right\rangle=\mu_{\Delta K}^{\Gamma} e^{-\alpha_{\Delta K}^{\Gamma}\left(E_{\nu}^{\prime}+E_{\nu}^{\prime \prime}\right)-\beta_{\Delta K}^{\Gamma}\left|E_{\nu}^{\prime}-E_{\nu}^{\prime \prime}\right|},
$$

where $E_{\nu}^{\prime}$ and $E_{\nu}^{\prime \prime}$ correspond to the vibrational energies without rotational contributions, as shown in Fig. 4 of Ref. 8. All irreducible representations display very close values for $\mu_{\Delta K}^{\Gamma}$, $\alpha_{\Delta K}^{\Gamma}$, and $\beta_{\Delta K}^{\Gamma}$ as shown by small dispersions around their averaged values computed over the lowest 150 vibrational eigenstates $(\nu=0, \ldots, 149)$ for $J=0, \ldots, 5$ and $K=0, \ldots, J$ for all $\Gamma_{i}^{ \pm}$irreducible representations as given in Table I ( $\mu$ in Debye and $\alpha$ and $\beta$ in $1 / \mathrm{cm}^{-1}$ ).

Based on the above results, we have obtained simpler expressions for $S_{n^{\prime \prime} n^{\prime}}$ and $L\left(\omega, \omega_{n^{\prime} n^{\prime \prime}}, \gamma\right)$ which are much easier to handle now. The other factors involved are already given by simple forms. After the first procedure, we apply the second procedure in which we introduce a new method to handle the summations over $n^{\prime \prime}$ and $n^{\prime}$ in Eq. (9). This method comes from the fact that one can reduce the original summations over two sets $n^{\prime \prime}$ and $n^{\prime}$ into much smaller summations which run over the two quantities $E_{n^{\prime \prime}}$ and $E_{n^{\prime}}$ because one can show that terms to be summed in Eq. (9) depend on the latter only. Then, we transform the latter sum- 
$K=1$
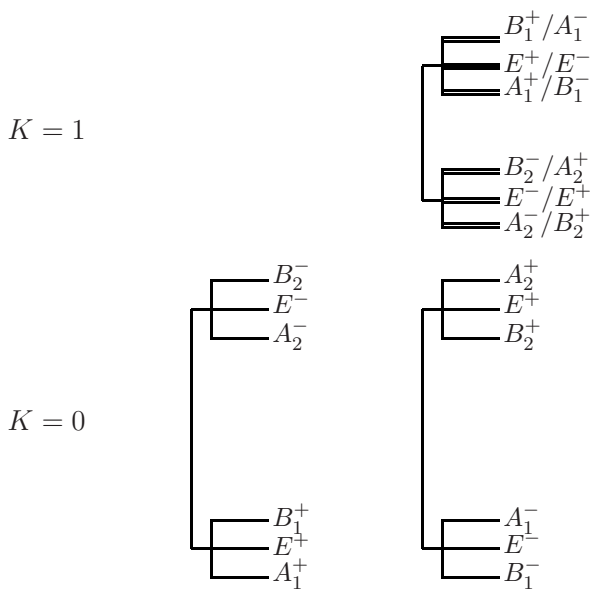

$J=0$

$J=1$

FIG. 1. The rotational energy level pattern associated with the rotational quantum numbers $J=0$ and 1 and $K=0$ and $|K|=1$.

mations into continuous integrations and evaluate them approximately. In the following, we explain this procedure step by step in detail.

\section{To reduce the original summations to simpler ones and to transform the latter into continuous integrations}

Because the dipole matrix elements do not depend on the rotational quantum numbers (except for the $\Delta K$ value), it would be helpful to separate summations over the rotation quantum numbers and over the vibrational quantum numbers such that

$$
\sum_{n} g_{n} e^{-E_{n} / k_{B} T}=\frac{1}{16} \sum_{\Gamma \nu} g_{\Gamma} e^{-E_{\nu} / k_{B} T} \sum_{J K} e^{-E_{J K} / k_{B} T} .
$$

The normalization factor (1/16) appearing in front of this equation is to comply with the standard convention, as used by Harris et al. ${ }^{19}$ in their definition of the monomer partition function $Q_{R V}^{M}(T)$, which affects the weights of $3 / 4$ and $1 / 4$ of ortho and para states, respectively. We note that the summation over the rotational quantum number $M$ is not present here because this summation is included in the HL factor. By taking a continuous limit for $E_{\nu}$, the summation over $\Gamma$ and $\nu$ can be transformed to an integral over $E_{\nu}$ such that

$$
\sum_{\Gamma \nu} f\left(E_{\nu}\right) \rightarrow \int d E_{\nu} \rho_{\nu}\left(E_{\nu}\right) f\left(E_{\nu}\right)
$$

where $\rho_{\nu}\left(E_{\nu}\right) d E_{\nu}$ is the number of vibrational states in the range from $E_{\nu}$ to $E_{\nu}+d E_{\nu}$. The distribution density $\rho_{\nu}\left(E_{\nu}\right)$ should correspond to the set of pure vibrational states $(J$ $=K=0$ ) considering all irreducible representations including their normalized nuclear weight $g_{\Gamma}$. However, due to the interplay between the symmetry representations $\Gamma$ and the allowed $J$ values as shown in Fig. $1,{ }^{8}$ one has to define this density from levels with $J=|K|=1$ to allow all irreducible representations to make contributions, and we have already shown that the different representations display very close values for the $\mu_{\Delta K}^{\Gamma}, \alpha_{\Delta K}^{\Gamma}$, and $\beta_{\Delta K}^{\Gamma}$ parameters.
The distribution density defined in this way should finally be divided by 2 because, as shown by the rotational energy level pattern (cf. Fig. 1), the $|K|=1$ notation actually means the two components $K=-1$ and $K=1$ which display the same energy levels (symmetric top approximation). This density $\rho_{\nu}\left(E_{\nu}\right)$ thus corresponds to a total normalized space and nuclear weight of 1 , encompassing all the states associated with a given $(J, K)$ pair of rotational quantum numbers. We have found a simple formula with which one can represent $\rho_{\nu}\left(E_{\nu}\right)$ well

$$
\rho_{\nu}\left(E_{\nu}\right)=D \times E_{\nu} e^{b E_{\nu}},
$$

where $D=2.17694 \times 10^{-4}$ (in $1 / \mathrm{cm}^{-2}$ ) and $b=2.59014$ $\times 10^{-3}$ (in $\left.1 / \mathrm{cm}^{-1}\right)$. A test of its applicability shows that

$$
\int_{0}^{1150 \mathrm{~cm}^{-1}} \rho_{\nu}\left(E_{\nu}\right) d E_{\nu}=1120 .
$$

The exact normalized number of states below $1150 \mathrm{~cm}^{-1}$ is 1114.

By introducing the distribution density $\rho_{\nu}\left(E_{\nu}\right)$ over the vibrational energy, one is able to rewrite the summation over the initial states as

$$
\sum_{n^{\prime \prime}} g_{n^{\prime \prime}} \rightarrow \sum_{J^{\prime \prime}=0} \sum_{K^{\prime \prime}=-J^{\prime \prime}}^{J^{\prime \prime}} \int d E_{\nu}^{\prime \prime} \rho_{\nu}\left(E_{\nu}^{\prime \prime}\right) .
$$

Similarly, we transform the summations over the rotational quantum numbers $J^{\prime \prime}$ and $K^{\prime \prime}$ into integrations over variables $j^{\prime \prime}$ and $k^{\prime \prime}$ with a uniform distribution density $\rho_{r}\left(j^{\prime \prime}, k^{\prime \prime}\right)=1$, which results from the fact that the degeneracy of the magnetic quantum number $M^{\prime \prime}$ has already been taken into account in the HL factor,

$$
\sum_{J^{\prime \prime}=0} \sum_{K^{\prime \prime}=-J^{\prime \prime}}^{J^{\prime \prime}} \rightarrow \int_{0}^{J_{\max }^{\prime \prime}} d j^{\prime \prime} \int_{-j^{\prime \prime}}^{j^{\prime \prime}} d k^{\prime \prime} .
$$

From any single initial state within a double fork characterized by the rotational parameters $j^{\prime \prime}$ and $k^{\prime \prime}$, there is only one possible transition to a given final allowed double fork state labeled by $J^{\prime}$ and $K^{\prime}$.

Thus, the summations over the initial and final states of the dimer can be expressed as

$$
\begin{aligned}
& \sum_{n^{\prime \prime}} \sum_{n^{\prime}>n^{\prime \prime}} g_{n^{\prime \prime}} \\
& \rightarrow \int_{0}^{J_{\max }^{\prime \prime}} d j^{\prime \prime} \int_{-j^{\prime \prime}}^{j^{\prime \prime}} d k^{\prime \prime} \sum_{\Delta J=0, \pm 1} \sum_{\Delta K=0, \pm 1} 16 \int d E_{\nu}^{\prime \prime} \rho_{\nu}\left(E_{\nu}^{\prime \prime}\right) \\
& \quad \times \int d E_{\nu}^{\prime} \rho_{\nu}\left(E_{\nu}^{\prime}\right)
\end{aligned}
$$

where $\Delta J=J^{\prime}-j^{\prime \prime}$ and $\Delta K=K^{\prime}-k^{\prime \prime}$. The extra factor of 16 comes from the fact that any given state, characterized by $\Gamma^{+/-}$, within a $\left(J^{\prime \prime}, K^{\prime \prime}\right)$ fork can make a transition to a $\Gamma^{-/+}$ state of another fork. The sum over all the $g_{\Gamma}$ 's [appearing in Eq. (13)] thus corresponds to 16 . With this transformation, one is able to rewrite Eq. (9) as 


$$
\begin{aligned}
& H(\omega, T)=\sum_{n^{\prime \prime}} \sum_{n^{\prime}>n^{\prime \prime}} \omega_{n^{\prime} n^{\prime \prime}} g_{n^{\prime \prime}} e^{-E_{n^{\prime \prime}} / k_{B} T}\left(1-e^{-\omega_{n^{\prime} n^{\prime \prime}} / k_{B} T}\right) \\
& \times S_{n^{\prime \prime} n^{\prime}} L\left(\omega, \omega_{n^{\prime} n^{\prime \prime}}, \gamma\right) \\
& =16 \int_{0}^{J_{\max }^{\prime \prime}} d j^{\prime \prime} \int_{-j^{\prime \prime}}^{j^{\prime \prime}} d k^{\prime \prime} \sum_{\Delta J=0, \pm 1} \sum_{\Delta K=0, \pm 1} \int d E_{\nu}^{\prime \prime} \rho_{\nu}\left(E_{\nu}^{\prime \prime}\right) \\
& \times \int d E_{\nu}^{\prime} \rho_{\nu}\left(E_{\nu}^{\prime}\right) \omega_{n^{\prime} n^{\prime \prime}}\left(e^{-E_{n^{\prime \prime}} / k_{B} T}-e^{-E_{n^{\prime}} / k_{B} T}\right) \\
& \times \mu_{\Delta K}^{2} e^{-2 \alpha_{\Delta K}\left(E_{\nu}^{\prime}+E_{\nu}^{\prime \prime}\right)} e^{-2 \beta_{\Delta K}\left|E_{\nu}^{\prime}-E_{\nu}^{\prime \prime}\right|} S\left(J^{\prime} K^{\prime} ; j^{\prime \prime} k^{\prime \prime}\right) \\
& \times\left[\delta\left(\omega-\omega_{n^{\prime} n^{\prime \prime}}\right)+\delta\left(\omega+\omega_{n^{\prime} n^{\prime \prime}}\right)\right] .
\end{aligned}
$$

We note that because we are only interested in $H(\omega, T)$ with $\omega \geq 0$ and the summations over $n^{\prime}$ and $n^{\prime \prime}$ in Eq. (20) have a limitation of $\omega_{n^{\prime} n^{\prime \prime}}>0$, the second $\delta$-function [i.e., $\delta(\omega$ $\left.\left.+\omega_{n^{\prime} n^{\prime \prime}}\right)\right]$ can be omitted in Eq. (20) because it does not make any contributions.

\section{To make further simplifications}

In order to benefit from the presence of the delta functions in the above expression, we introduce two new integral variables $E \equiv E_{\nu}^{\prime}+E_{\nu}^{\prime \prime}$ and $\omega_{n^{\prime} n^{\prime \prime}} \equiv E_{\nu}^{\prime}-E_{\nu}^{\prime \prime}+\Delta E_{R}$, where $\Delta E_{R}$ $=E_{R}^{\prime}-E_{R}^{\prime \prime}$ corresponds to the change in rotational energy, to replace $E_{\nu}^{\prime \prime}$ and $E_{\nu}^{\prime}$,

$$
\begin{aligned}
E_{R}(j, k) & =B j(j+1)+A k^{2} \Rightarrow \Delta E_{R}(j, k) \\
& \approx 2 B j \times \Delta j+2 A k \times \Delta k
\end{aligned}
$$

in the limit of large $j$ and $k$ values. The Jacobian of this variable change $\left(E_{\nu}^{\prime}, E_{\nu}^{\prime \prime}\right) \rightarrow\left(E, \omega_{n^{\prime} n^{\prime \prime}}\right)$ is $1 / 2$. In terms of these two variables, the product of the densities $\rho_{\nu}\left(E_{\nu}^{\prime \prime}\right) \rho_{\nu}\left(E_{\nu}^{\prime}\right)[$ see Eq. (15)] can be expressed as

$$
\begin{aligned}
\rho_{\nu}\left(E_{\nu}^{\prime \prime}\right) \rho_{\nu}\left(E_{\nu}^{\prime}\right) & =\frac{D^{2}}{4}\left[E^{2}-\left(\omega_{n^{\prime} n^{\prime \prime}}-\Delta E_{R}\right)^{2}\right] e^{b E} \\
& \approx \frac{D^{2}}{4}\left[E^{2}-\omega_{n^{\prime} n^{\prime \prime}}^{2}\right] e^{b E}
\end{aligned}
$$

where we have made the approximation $\Delta E_{R} \ll E, \omega_{n^{\prime} n^{\prime \prime}}$, which is valid except in the low-frequency domain. Similarly, using $E_{n^{\prime \prime}}=\frac{1}{2}\left(E+\Delta E_{R}-\omega_{n^{\prime} n^{\prime \prime}}\right)+E_{R}^{\prime \prime}$, one can rewrite

$$
\begin{aligned}
& \omega_{n^{\prime} n^{\prime \prime}}\left(e^{-E_{n^{\prime \prime}} / k_{B} T}-e^{-E_{n^{\prime}} / k_{B} T}\right) \\
& \quad=2 \omega_{n^{\prime} n^{\prime \prime}} \sinh \left(\omega_{n^{\prime} n^{\prime \prime}} / 2 k_{B} T\right) \times e^{-E_{R^{\prime \prime}}^{\prime \prime} / k_{B} T} e^{-\left(E+\Delta E_{R}\right) / 2 k_{B} T}
\end{aligned}
$$

and

$$
\mu_{\Delta K}^{2} e^{-2 \alpha_{\Delta K}\left(E_{\nu}^{\prime}+E_{\nu}^{\prime \prime}\right)-2 \beta_{\Delta K}\left|E_{\nu}^{\prime}-E_{\nu}^{\prime \prime}\right|}=\mu_{\Delta K}^{2} e^{-2 \alpha_{\Delta K} E-2 \beta_{\Delta K}\left|\omega_{n^{\prime} n^{\prime \prime}}-\Delta E_{R}\right|} .
$$

Then, one can easily carry out the integration over $\omega_{n^{\prime} n^{\prime \prime}}$ due to a presence of the delta functions and rewrite Eq. (20) as
TABLE II. Limiting values of the HL factor $S\left(J^{\prime} K^{\prime} ; j k\right)$ when $j \gg k$.

\begin{tabular}{lrr}
\hline \hline$S\left(J^{\prime} K^{\prime} ; j k\right)$ & $\Delta K=0$ & $\Delta K= \pm 1$ \\
\hline$\Delta J=-1$ & $\approx j$ & $\approx(j-1) / 4$ \\
$\Delta J=0$ & $\approx 0$ & $\approx(2 j-1) / 4$ \\
$\Delta J=+1$ & $\approx j+1$ & $\approx(j+2) / 4$ \\
\hline \hline & & \\
& $\times \sum_{\Delta J=0, \pm 1 \Delta K=0, \pm 1} e^{-E_{R}^{\prime \prime} / k_{B} T} e^{\Delta E_{R}\left(2 \beta_{\Delta K}-1 / 2 k_{B} T\right)} e^{-2 \beta_{\Delta K} \omega}$ \\
& $\times \mu_{\Delta K^{2}}^{2} S\left(J^{\prime} K^{\prime} ; j^{\prime \prime} k^{\prime \prime}\right)$ & \\
& $\times \int_{E_{\min }}^{\infty}\left[\frac{\omega}{2 k_{B} T}\right) \int_{0}^{J_{\max }^{\prime \prime}} d j^{\prime \prime} \int_{-j^{\prime \prime}}^{j^{\prime \prime}} d k^{\prime \prime}$ &
\end{tabular}

In deriving the above expression, we have assumed that $\mid \omega$ $-\Delta E_{R} \mid=\omega-\Delta E_{R}$. This assumption is valid as long as $\omega$ $>\Delta E_{R}$, which again does not apply to the very lowfrequency domain. In the above integration over $E$, as $\left(E^{2}\right.$ $\left.-\omega^{2}\right) \propto E_{\nu}^{\prime} \times E_{\nu}^{\prime \prime}$ has to be positive, the integration starts from $E_{\min }=\omega$.

\section{To carry out the integration over $\mathcal{E}$ analytically}

In order to evaluate Eq. (25), we introduce a parameter $\eta_{\Delta K}$ defined by

$$
\eta_{\Delta K} \equiv-\left(b-2 \alpha_{\Delta K}^{\Gamma}-1 / 2 k_{B} T\right) .
$$

This parameter depends on the temperature and its value (in units of $\left.1 / \mathrm{cm}^{-1}\right)$ is positive. As an example, $\eta_{\Delta K}(T$ $=300 \mathrm{~K})=4.670 \times 10^{-3}\left(\right.$ in $\left.1 / \mathrm{cm}^{-1}\right)$. By carrying out the integration over $\mathcal{E}$ analytically, one finally obtains

$$
\int_{\omega}^{\infty} d E\left(E^{2}-\omega^{2}\right) e^{-\eta_{\Delta K} E}=\frac{2 e^{-\eta_{\Delta K} \omega}}{\eta_{\Delta K}^{3}}\left(\eta_{\Delta K} \omega+1\right),
$$

which leads to the expression for $H(\omega, T)$,

$$
\begin{aligned}
H(\omega, T)= & 8 D^{2} \omega \sinh \left(\frac{\omega}{2 k_{B} T}\right) \int_{0}^{J_{\max }^{\prime \prime}} d j^{\prime \prime} \int_{-j^{\prime \prime}}^{j^{\prime \prime}} d k^{\prime \prime} \\
& \times \sum_{\Delta J=0, \pm 1} \sum_{\Delta K=0, \pm 1} e^{-E_{R^{\prime \prime} / k_{B} T}} \\
& \times e^{\left(2 \beta_{\Delta K}-1 / 2 k_{B} T\right) \Delta E_{R}} e^{-\left(2 \beta_{\Delta K^{+}}+\eta_{\Delta K}\right) \omega} \\
& \times \frac{\mu_{\Delta K}^{2}}{\eta_{\Delta K}^{3}}\left(1+\eta_{\Delta K} \omega\right) S\left(J^{\prime} K^{\prime} ; j^{\prime \prime} k^{\prime \prime}\right) .
\end{aligned}
$$

\section{Summation over $\Delta J$ with $\Delta K$ fixed}

Let us consider the HL factors $S\left(J^{\prime} K^{\prime}, j^{\prime \prime} k^{\prime \prime}\right)$. On can show that in the limit of $j^{\prime \prime} \gg k^{\prime \prime}$, the following approximate expressions shown in Table II are applicable. ${ }^{20}$ We first consider the summation over $\Delta J$ with fixed $\Delta K$. This summation can be restricted to the terms 


$$
\xi_{\Delta K}=\sum_{\Delta J=0, \pm 1} e^{\gamma_{\Delta K} \Delta E_{R} S\left(J^{\prime} K^{\prime} ; j^{\prime \prime} k^{\prime \prime}\right)}
$$

where we have defined the parameter $\gamma_{\Delta K}=2 \beta_{\Delta K}-1 / 2 k_{B} T$, which allows us to rewrite the $H(\omega, T)$ term [i.e., Eq. (25)] as

$$
\begin{aligned}
H(\omega, T)= & 8 D^{2} \omega \sinh \left(\frac{\omega}{2 k_{B} T}\right) \int_{0}^{J_{\max }^{\prime \prime}} d j^{\prime \prime} \int_{-j^{\prime \prime}}^{j^{\prime \prime}} d k^{\prime \prime} \\
& \times \sum_{\Delta K=0, \pm 1} e^{-E_{R}^{\prime \prime} / k_{B} T} e^{-\left(2 \beta_{\Delta K^{+}} \eta_{\Delta K}\right) \omega} \\
& \times \frac{\mu_{\Delta K}^{2}}{\eta_{\Delta K}^{3}}\left(1+\eta_{\Delta K} \omega\right) \xi_{\Delta K} .
\end{aligned}
$$

For the sum over $\Delta K=0$, the $\Delta J=0$ contribution can be left out due to the zero value of the HL factor, and using the asymptotic expressions for $\Delta E_{R}$ and the HL factors,

$$
\xi_{0}=j\left(e^{+2 B \gamma_{0} j}+e^{-2 B \gamma_{0} j}\right) .
$$

The sums over $\Delta K= \pm 1$ are

$$
\xi_{ \pm 1}=\frac{j}{4} e^{ \pm 2 A \gamma_{1} k}\left(e^{+2 B \gamma_{1} j}+2+e^{-2 B \gamma_{1} j}\right) .
$$

\section{Integration over $\boldsymbol{j}$ and $k$}

Consider now the double integration over $j$ and $k$,

$$
\Xi_{\Delta K}=\int_{0}^{J_{\max }^{\prime \prime}} d j^{\prime \prime} \int_{-j^{\prime \prime}}^{j^{\prime \prime}} d k^{\prime \prime} e^{-E_{R}^{\prime \prime} k_{B} T} \xi_{\Delta K}
$$

where the rotational energy $E_{R}$ has been given in Eq. (21). Because values of the integrand of Eq. (33) decrease very quickly as $k$ increases, one can extend $-j^{\prime \prime}$ and $j^{\prime \prime}$, the lower and upper limits of the integration over $k^{\prime \prime}$, to $-\infty$ and $\infty$, respectively. Similarly, one can extend $J_{\max }^{\prime \prime}$, the upper limit of the integration over $j^{\prime \prime}$, to $\infty$ too. Then, one is able to carry out these integrations analytically such that

$$
\begin{aligned}
\Xi_{0} & =\int_{-\infty}^{+\infty} d k \int_{0}^{\infty} d j \xi_{0} e^{-E_{R}^{\prime \prime} / k_{B} T}=\sqrt{\frac{\pi\left(k_{B} T\right)^{3 / 2}}{A B^{2}}}, \\
\Xi_{ \pm 1} & =\int_{-\infty}^{+\infty} d k \int_{0}^{\infty} d j \xi_{ \pm 1} e^{-E_{R}^{\prime \prime} / k_{B} T} \\
& =\frac{e^{A \gamma_{1}^{2} k_{B} T}}{2} \times \sqrt{\frac{\pi\left(k_{B} T\right)^{3 / 2}}{A B^{2}}},
\end{aligned}
$$

and to obtain the final expression

$$
\begin{aligned}
\tilde{H}(\omega, T)= & 8 D^{2} \omega \sinh \left(\frac{\omega}{2 k_{B} T}\right) \sqrt{\frac{\pi\left(k_{B} T\right)^{3 / 2}}{A B^{2}}} \\
& \times \sum_{\Delta K=0,1}\left[\delta_{\Delta K 0}+\delta_{\Delta K 1} e^{A \gamma_{\Delta K}^{2} k_{B} T}\right] \\
& \times e^{-\left(2 \beta_{\Delta K^{+}} \eta_{\Delta K}\right) \omega} \frac{\mu_{\Delta K}^{2}}{\eta_{\Delta K}^{3}}\left(1+\eta_{\Delta K} \omega\right),
\end{aligned}
$$

where $\delta_{\triangle K 0}$ and $\delta_{\triangle K 1}$ are the Kronecker delta.

One has finally to untangle the $T$ dependence of the sum in the above expression since temperature appears in both

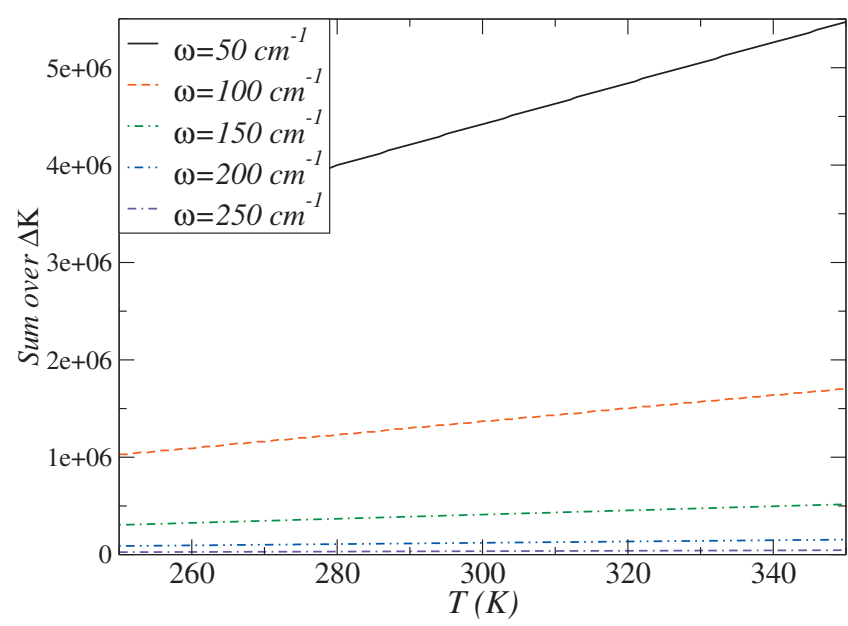

FIG. 2. Variation with temperature of the sum term appearing in Eq. (35), for different frequencies $\omega_{0}=50,100,150,200$, and $250 \mathrm{~cm}^{-1}$, over the range $T=200-400 \mathrm{~K}$. The $T$ dependence of each curve was obtained from a fit to the form $S\left(\omega_{0}\right) T^{n}$ which leads to $n$ values 1.436, 1.487, 1.554, 1.633, and 1.717 , respectively.

$\eta_{\Delta K}$ and $\gamma_{\Delta K}$. A direct determination by inspection is difficult as the temperature factor is mixed with constants or frequency $\omega$. For example, Eq. (26) which defines $\eta_{\Delta K}$ shows that its $T$ dependence varies from $T^{-1}$ (for $T \rightarrow 0$ ) to $T^{0}$ (for $T \rightarrow \infty)$. Fitting these quantities over the range $T$ $=200-400 \mathrm{~K}$ actually leads to dependence as $T^{-0.516}$ for $\eta_{\Delta K=0}$ and $T^{-0.694}$ for $\eta_{\Delta K=1}$. In Fig. 2, we present values of the sum in Eq. (35) over the temperature range $T$ $=200-400 \mathrm{~K}$ for frequencies $\omega_{0}=50,100,150,200$, and $250 \mathrm{~cm}^{-1}$ respectively. A fit of these curves, shown in Fig. 2, to the expression $S\left(\omega_{0}\right) T^{n}$ leads to exponent values $n=1.436,1.487,1.554,1.633$, and 1.717 , respectively. We thus made the approximation of a simplified $T$ dependence of the form $T^{3 / 2}$ to represent the behavior of this sum in the low-frequency regime.

\section{Conclusions on the $T$ dependence of the dimer absorption}

By combining results obtained from the above sections, we can draw a basic conclusion that the $T$ dependence of the dimer absorption coefficient can be well described as

$$
\begin{gathered}
\alpha(\omega, T) \propto n_{M} P_{M} \times T^{-11 / 2} e^{D_{0} / k_{B} T} \times \sinh \left(\frac{\omega}{2 k_{B} T}\right) \times T^{3 / 2} \times T^{3 / 2} \\
=n_{M} P_{M} \times \frac{e^{D_{0} / k_{B} T}}{T^{5 / 2}} \times \sinh \left(\frac{\omega}{2 k_{B} T}\right) .
\end{gathered}
$$

In the case where $\hbar \omega / 2 k_{B} T \ll 1$, the dependences of $\alpha(\omega, T)$ on the frequency $\omega$ and the temperature $T$ are separable. As a result, the $T$ dependence of $\alpha(\omega, T)$ becomes uniform for the frequency $\omega$ and it can be expressed as

$$
\alpha(\omega, T) \propto n_{M} P_{M} \times \frac{e^{D_{0} / k_{B} T}}{T^{7 / 2}},
$$

where $n_{M} P_{M}$ may contribute an extra $T$ dependence. More specifically, if the units selected for $\alpha(\omega)$ are $\mathrm{cm}^{2}$ molecule $\mathrm{atm}^{-1}, n_{M} P_{M}$ would be independent of the temperature. If the units are $\mathrm{dB} / \mathrm{km}(\mathrm{kPa})^{-2}, n_{M} P_{M}$ would 


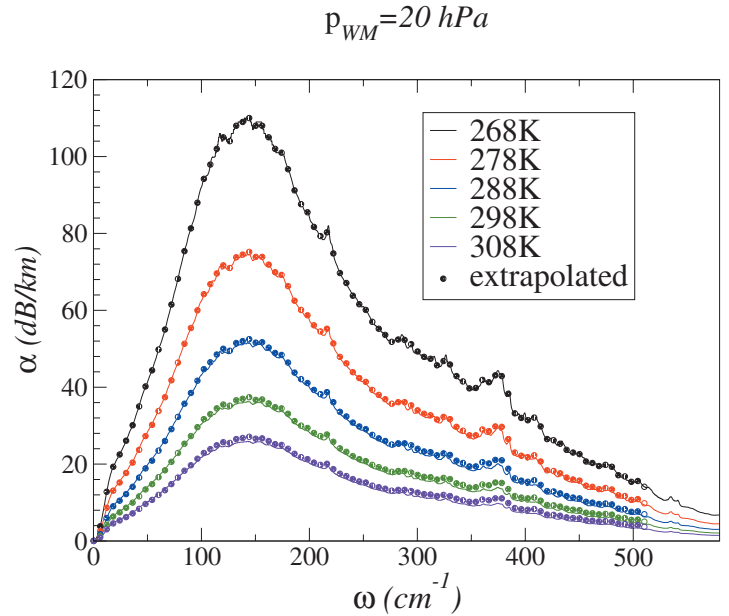

FIG. 3. Comparison between the exactly computed absorption coefficient $\alpha(\omega, T)$ [i.e., Eq. (1)] for five different temperatures in the range 268-308 K, represented by full curves, and the extrapolated values $\alpha_{\text {ext }}(\omega, T)$ by means of Eq. (38), represented by dots, using $\alpha(\omega, 268 \mathrm{~K})$ as the reference curve. One can note that the extrapolated values are quasi-indistinguishable from the original ones even though the coefficient changes noticeably over this temperature range.

contribute an extra factor of $T^{-1}$ in Eqs. (36) and (37).

It is worth mentioning that Eqs. (36) and (37) are the most important results in our study of the $T$ dependence of the dimer absorption. In contrast with the other two mechanisms (i.e., the far-wing theory and CIA), the $T$ dependence of the dimer theory can be well described by a simple analytic form. This indicates that in comparisons with the other two mechanisms, the pattern of its $T$ dependence is much simpler. This feature is especially true for low-frequency regions as mentioned above.

\section{E. Test of the validity of the model}

In order to test the validity of the model proposed above [Eq. (36)], we performed two series of tests.

\section{Extrapolation of results obtained at $T=268 \mathrm{~K}$}

The first test consists of starting from the absorption coefficient $\alpha\left(\omega, T_{r e f}\right)$ (in $\left.\mathrm{dB} / \mathrm{km}\right)$ exactly computed from Eq. (1), to extrapolate these values to a different temperature $T_{\text {ext }}$ according to the $T$ dependence found above, i.e., by rescaling the $\alpha\left(\omega, T_{\text {ref }}\right)$ curve as described below,

$$
\alpha_{\mathrm{ext}}(\omega, T)=\frac{e^{D_{0} / k_{B} T}}{e^{D_{0} / k_{B} T_{\mathrm{ref}}}} \times \frac{T_{\mathrm{ref}}^{9 / 2}}{T^{9 / 2}} \times \alpha\left(\omega, T_{\mathrm{ref}}\right) .
$$

The results are shown in Fig. 3 where the full curves correspond to the exact values [i.e., Eq. (1)] and the extrapolated values derived with $D_{0}=1771 \mathrm{~K}$ appear as dots superimposed on these curves. One can check that the extrapolated curves are quasi-indistinguishable from the exactly computed values [i.e., Eq. (1)], which validates the $T$ dependence of $e^{D_{0} / k_{B} T} \times T^{-9 / 2}$ found previously.

\section{Direct calculation from the model}

A more stringent test consists in directly computing the absorption coefficient $\alpha(\omega, T)$ from the model expression for

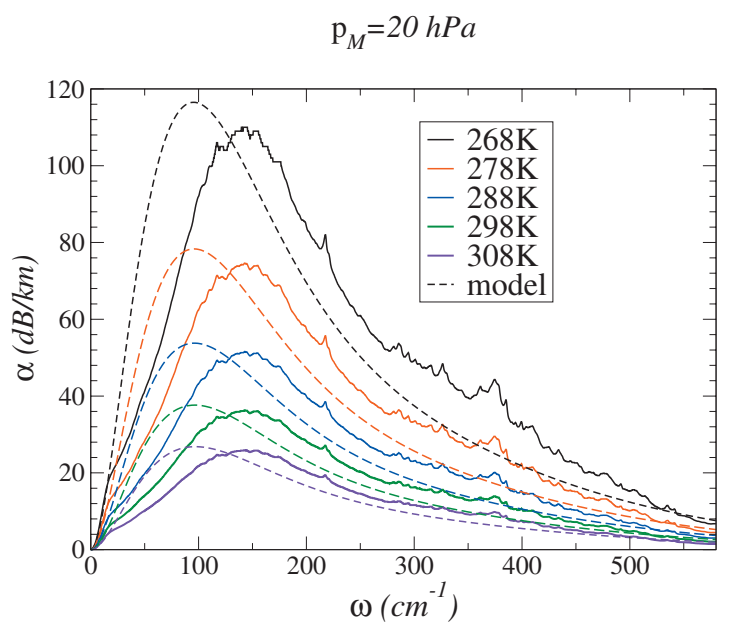

FIG. 4. Comparison between the exactly computed absorption coefficient $\alpha(\omega, T)$ [i.e., Eq. (1)] for five different temperatures in the range 268-308 K, represented by full curves, and the approximated values $\widetilde{\alpha}(\omega, T)$ obtained by means of Eq. (39), depicted by dashed lines. In this latter equation, $\widetilde{H}(\omega, T)$ represents the model expression [i.e., Eq. (35)] of the exact function $H(\omega, T)$ [i.e., Eq. (9)]

$H(\omega, T)$ which has been derived in Sec. II C. Starting from Eq. (1) and introducing the successive manipulations [i.e., Eqs. (3), (6), and (7)] and an approximate expression $\widetilde{H}(\omega, T)$ defined by Eq. (35), one finally expresses the approximate absorption coefficient $\widetilde{\alpha}(\omega, T)$ in terms of simple parameters characterizing the water dimer [viz. rotational constants $A$ and $B$, density of vibrational states $\rho_{v}\left(E_{v}\right)$ and transition dipole functions $\left.\mu_{\Delta K}\left(E_{v}^{\prime \prime}, E_{v}^{\prime}\right)\right]$

$$
\widetilde{\alpha}(\omega, T)=\frac{4 \pi^{2} h^{3}}{3 \hbar c} \cdot \frac{p_{M}^{2}}{\left(k_{B} T\right)^{2}} \cdot \frac{e^{D_{0} / k_{B} T}}{\left(\pi m_{M} k_{B} T\right)^{3 / 2}\left(Q_{R}^{M}\right)^{2}} \tilde{H}(\omega, T) .
$$

The results are plotted in Fig. 4 together with those from our exact calculations [i.e., Eq. (1)]. One can remark that Eq. (35) gives a reasonably correct description of the absorption band in the far infrared region, except that the maximum is somewhat red shifted, but the intensity maxima and the integrated absorptions are well reproduced for these different temperatures. This overall agreement is remarkable if one considers the different approximations invoked to simplify the expression of the absorption coefficient. Among them, one can cite the following.

(i) All irreducible representations have been handled in a similar way, through averaging of the total vibrational density of states [i.e., Eq. (17)] although the doubly degenerate $E^{ \pm}$states display a different pattern (see Fig. 1).

(ii) Rotation has been fully decoupled from vibration. However, the true repartition of $(J, K)$-rotational states, displayed in Fig. 1, reveals that the splitting between the rotational forks actually depends on the vibrational state considered. Also, in our exact calculations, we made the $A$ and $B$ rotational constants depend on vibrational energy $E$, e.g., $A_{v J K}^{\Gamma} \cong a_{J K}^{\Gamma}+b_{J K}^{\Gamma}$ $\times E_{v}$, to take into account the centrifugal distortion. 
(iii) The simple interpolation formula
$\mu_{\Delta K}^{\Gamma} e^{-\alpha_{\Delta K}^{\Gamma}\left(E_{v}^{\prime}+E_{v}^{\prime \prime}\right)} e^{-\beta_{\Delta K}^{\Gamma}\left|E_{v}^{\prime}-E_{v}^{\prime \prime}\right|}$ has been used to represent the dipole matrix elements $\left\langle\Phi_{v^{\prime} K^{\prime}}^{J^{\prime} \Gamma^{\prime}}\left|\mu_{\Delta K}^{B F}\right| \Phi_{v^{\prime \prime} K^{\prime \prime}}^{J^{\prime \prime} \Gamma^{\prime \prime}}\right\rangle$, whereas their exactly computed values were used for states up to $600 \mathrm{~cm}^{-1}$ of vibrational excitation.

\section{CIA FOR THE $\mathrm{H}_{2} \mathrm{O}-\mathrm{H}_{2} \mathrm{O}$ PAIR}

It is well known that during collisions of two interacting molecules, transient dipole moments occur and the latter can cause absorptions in the same way as permanent dipole moments do. ${ }^{13}$ In general, the expression for the collisioninduced dipole for the polyatomic molecular pair is given by $^{21}$

$$
\mu_{1 \nu}=\sum_{c} \mu_{1 \nu}^{(c)}=\sum_{c} A^{(c)}\left(R, r_{1 N}, r_{2 N}\right) \psi_{1 \nu}^{(c)}\left(\Omega_{1}, \Omega_{2}, \Omega\right),
$$

where $r_{i N}$ are the vibrational coordinates of molecule $i, R$ is the distance between the centers of mass of the two molecules, $\Omega$ is the orientation of $R$, and $\Omega_{1}$ and $\Omega_{2}$ specify the orientations for the two molecules, respectively. In Eq. (40), (c) is a shorthand notation for the set of numbers $\left(\lambda_{1}, \lambda_{2}, L, \Lambda ; \nu_{1}, \nu_{2}\right)$ specifying the dipole components, $A^{(c)}$ are coefficients, and the quantities $\psi_{1 \nu}^{(c)}$ are given by

$$
\begin{aligned}
\psi_{1 \nu}^{(c)}= & \sqrt{\frac{4 \pi\left(2 \lambda_{1}+1\right)\left(2 \lambda_{2}+1\right)}{3}} \sum_{m, M} C(\Lambda L 1, m M \nu) \\
& \times \sum_{m_{1}, m_{2}} C\left(\lambda_{1} \lambda_{2} \Lambda, m_{1} m_{2} m\right) \\
& \times D_{m_{1} k_{1}}^{\lambda_{1}}\left(\Omega_{1}\right)^{*} D_{m_{2} k_{2}}^{\lambda_{2}}\left(\Omega_{2}\right)^{*} Y_{L M}(\Omega) .
\end{aligned}
$$

Here, the $C$ 's are Clebsch-Gordan factors, the $D$ 's are rotation matrices, and $Y_{L M}$ are spherical harmonics.

\section{A. Main component of dipole-induced dipole for the $\mathrm{H}_{2} \mathrm{O}-\mathrm{H}_{2} \mathrm{O}$ pair}

For simplicity, in the present study we consider only the main contribution (i.e., dipole-induced dipole through the isotropic part of the polarizability tensor) to the CIA for the $\mathrm{H}_{2} \mathrm{O}-\mathrm{H}_{2} \mathrm{O}$ pair. This mechanism corresponds to a specified component of $\mu_{1 \nu}$ with $\lambda_{1}=1, \lambda_{2}=0, \Lambda=1$, and $L=2$ which is labeled as $\mu_{1 \nu}^{\left(c_{0}\right)}$ later. The $\mathrm{H}_{2} \mathrm{O}$ molecule belongs to the $C_{2 v}$ point group which means that there is a symmetry resulting from a $180^{\circ}$ rotation around the symmetry axis of the molecule and two plane reflections passing through this axis. It is worth mentioning that the dipole operator $\mu_{1 v}$ must be invariant when any element of the molecular symmetry group which consists two operations I (an identity operator) and $C_{2}$ (a rotation around the symmetry axis with $180^{\circ}$ ) is applied to each of these two $\mathrm{H}_{2} \mathrm{O}$ molecules. ${ }^{21-23}$ However, after the $C_{2}$ operation is performed, the rotation matrix $D_{m_{i} k_{i}}^{\lambda_{i}}\left(\Omega_{i}\right)^{*}$ is transformed into $(-1)^{k_{i}} D_{m_{i} k_{i}}^{\lambda_{i}}\left(\Omega_{i}\right)^{*}$. This implies that $k_{i}$ must be an even number. By imposing the symmetry requirement mentioned above on this main component, one can conclude that the associated factors $D_{m_{1} k_{1}}^{1}\left(\Omega_{1}\right)^{*}$ and $D_{m_{2} k_{2}}^{0}\left(\Omega_{2}\right)^{*}$ must be replaced by $\sqrt{4 \pi / 3} Y_{1 m_{1}}\left(\Omega_{1}\right)$ and 1 , respectively. (For terms with higher $\lambda_{i}$ values, one has to figure out detailed expressions $\Phi_{\lambda_{i} m_{i}}\left(\Omega_{i}\right)$ used to replace $D_{m_{i} k_{i}}^{\lambda_{i}}\left(\Omega_{i}\right)^{*}$. In general, $\Phi_{\lambda_{i} m_{i}}\left(\Omega_{i}\right)$ are linear combinations of $D_{m_{i} k_{i}^{\prime}}^{\lambda_{i}{ }^{\prime} k_{i}}\left(\Omega_{i}\right)^{*}$ with different $k_{i}^{\prime}$, which are invariant under the symmetry group operations.) Therefore, the component $\mu_{1 \nu}^{\left(c_{0}\right)}$ can be expressed as

$$
\begin{aligned}
\psi_{1 \nu}^{\left(c_{0}\right)}= & \frac{4 \pi}{\sqrt{3}} \sum_{M, m_{1}} C\left(121, m_{1} M \nu\right) C\left(101, m_{1} 0 m_{1}\right) \\
& \times Y_{1 m_{1}}\left(\Omega_{1}\right) Y_{2 M}(\Omega) \\
= & \frac{4 \pi}{\sqrt{3}} \sum_{m_{1}} C\left(121, m_{1} \nu-m_{1} \nu\right) Y_{1 m_{1}}\left(\Omega_{1}\right) Y_{2 \nu-m_{1}}(\Omega) .
\end{aligned}
$$

This implies that by making proper replacements, formulas developed for a pair of linear molecules are also applicable here. For this component, the corresponding coefficient $A^{\left(c_{0}\right)}\left(R, r_{1 N}, r_{2 N}\right)$ in Eq. (40) is given by ${ }^{13}$

$$
A^{\left(c_{0}\right)}\left(R, r_{1 N}, r_{2 N}\right)=-\frac{\sqrt{2} \mu\left(r_{1 N}\right) \alpha\left(r_{2 N}\right)}{R^{3}},
$$

where $\mu\left(r_{1 N}\right)$ is the dipole moment and $\alpha\left(r_{2 N}\right)$ is the isotropic part of the polarizability tensor.

\section{B. CIA spectrum}

Because CIA occurs for interacting pairs of molecules, the intensity of each component and the corresponding integrated intensity are proportional to the number density of the pair. In the literature, researchers usually choose $\mathrm{cm}^{-1} /$ amagat $^{2}$ as the units for the CIA coefficient $\alpha(\omega)$. In the following, we follow the same choice unless we state otherwise. We note that in terms of the number density of monomers $\rho_{\mathrm{H}_{2} \mathrm{O}}$, the number density of the $\mathrm{H}_{2} \mathrm{O}-\mathrm{H}_{2} \mathrm{O}$ pair is $\rho_{\mathrm{H}_{2} \mathrm{O}}^{2} / 2$. However, for the mechanism considered here, there are two possibilities by interchanging the roles between the two $\mathrm{H}_{2} \mathrm{O}$ molecules. In other words, where dipole-induced dipole through the isotropic part of the polarizability tensor there are two possibilities by switching roles of the two $\mathrm{H}_{2} \mathrm{O}$ molecules: one with $\lambda_{1}=1, \lambda_{2}=0, \Lambda=1, L=2$, and the other with $\lambda_{1}=0, \lambda_{2}=1, \Lambda=1, L=2$. Therefore, the effective pair number density associated with this mechanism is $\rho_{\mathrm{H}_{2} \mathrm{O}}^{2}$. (We note that one has to be careful in treating bands such as the $v_{1}$, which is both Raman and IR allowed because one can have vibration-rotational transitions arising from the polarizability.) Then, the absorption coefficient $\alpha(\omega)$ can be written as

$$
\alpha(\omega)=\rho_{\mathrm{H}_{2} \mathrm{O}}^{2} \omega\left(1-e^{-\hbar \omega / k_{B} T}\right) F(\omega),
$$

where $F(\omega)$ is the spectral density which satisfies the detailed balance principle,

$$
F(-\omega)=e^{-\hbar \omega / k_{B} T} F(\omega) .
$$

In addition, the spectral density can be expressed as ${ }^{24}$

$$
F(\omega)=\frac{4 \pi^{2}}{3} \alpha_{F} n_{L}^{2} a_{0}^{5} \sum_{i_{1} f_{1}} \sum_{i_{2} f_{2}} S_{\left(n_{0}\right)}(T) G\left(\omega-\omega_{i_{1} f_{1}}-\omega_{i_{2} f_{2}}\right),
$$

where dimensionless $\alpha_{F}$ is the fine structure constant, $n_{L}\left(=2.6868 \times 10^{19}\right.$ molecule $\left./ \mathrm{cm}^{3}\right)$ is the number density of 
the gas at standard temperature and pressure, and $a_{0}$ (in $\mathrm{cm}$ ) is the Bohr radius. In Eq. (46), $G(\omega)$ is a normalized line shape function and $\omega_{i f}=\left(E_{f}-E_{i}\right) / \hbar$, where $E_{f}$ and $E_{i}$ are energies of the $\mathrm{H}_{2} \mathrm{O}$ molecule in its final state $f$ and initial state $i$. Because the isotropic part of the polarizabilty does not lead to rotational transitions, one can assume that $i_{2}=f_{2}$ and $\omega_{i_{2} f_{2}}=0$. We note that in deriving Eq. (46) for $F(\omega)$, the strength $S_{\left(n_{0}\right)}(T)$ whose explicit expression is given later by Eq. (48) is a dimensionless number because its dimensions have already been taken into account by introducing $\alpha_{F}$ and $a_{0}{ }^{5}$ and evaluating the matrix elements $\left\langle B(R)^{2}\right\rangle$ in atomic units.

At this stage, we would like to extend an expression for the strength $S_{\left(n_{0}\right)}(T)$ applicable for a pair of the linear molecules to a pair of the $\mathrm{H}_{2} \mathrm{O}$ molecules. We note that this extension is only valid for the main component $\left(c_{0}\right)$ of interest here. For later convenience, one introduces a new notation $B_{\left(n_{0}\right)}(R)$ which is defined as the matrix element of $A^{\left(c_{0}\right)}\left(R, r_{1 N}, r_{2 N}\right)$ between the initial and final vibrational states of the molecular pair by

$$
B_{\left(n_{0}\right)}(R)=\left\langle v_{1} v_{2} J_{1} J_{2}\left|A^{\left(c_{0}\right)}\left(R, r_{1 N}, r_{2 N}\right)\right| v_{1}^{\prime} v_{2}^{\prime} J_{1}^{\prime} J_{2}^{\prime}\right\rangle,
$$

where the subscript $\left(n_{0}\right)$ is a shorthand notation for $\left\{v_{1} v_{2} J_{1} J_{2} v_{1}^{\prime} v_{2}^{\prime} J_{1}^{\prime} J_{2}^{\prime}\right\}$ and $\left(c_{0}\right)$. Then, one can adopt an expression for the strength $S_{\left(n_{0}\right)}(T)$ applicable for the linear molecule pair, $^{24}$

$$
S_{\left(n_{0}\right)}(T)=P_{J_{1}} C\left(J_{1} 1 J_{1}^{\prime}, 000\right)^{2} P_{J_{2}} C\left(J_{2} 0 J_{2}^{\prime}, 000\right)^{2}\left\langle B_{\left(n_{0}\right)}(R)^{2}\right\rangle,
$$

where $P_{J}(T)$ are normalized Boltzmann factors. In the above expression, $\langle\cdots\rangle$ is a simple notation for

$$
\langle f(R)\rangle=4 \pi \int_{0}^{\infty} f(R) g(R) R^{2} d R,
$$

where $g(R)$ is the pair correlation function at low density and can be approximately expressed as

$$
g(R)=e^{-V_{\text {iso }}(R) / k_{B} T} .
$$

With Eqs. (43), (47), and (49), one can derive an explicit expression for $\left\langle B_{\left(n_{0}\right)}(R)^{2}\right\rangle$ as

$$
\begin{aligned}
\left\langle B_{\left(n_{0}\right)}(R)^{2}\right\rangle= & 2\left\langle v_{1} J_{1}\left|\mu\left(r_{1 N}\right)\right| v_{1}^{\prime} J_{1}^{\prime}\right\rangle^{2} \\
& \times\left\langle v_{2} J_{2}\left|\alpha\left(r_{2 N}\right)\right| v_{2}^{\prime} J_{2}^{\prime}\right\rangle^{2} I(T),
\end{aligned}
$$

where $I(T)$ is a temperature-dependent integral defined by

$$
I(T)=4 \pi \int_{0}^{\infty} \frac{1}{R^{6}} e^{-V_{i s o}(R) / k_{B} T} R^{2} d R .
$$

At this stage, we wish to simplify derivation of $S_{\left(n_{0}\right)}(T)$ further. With respect to the second $\mathrm{H}_{2} \mathrm{O}$ molecule, one can replace $C\left(J_{2} 0 J_{2}^{\prime}, 000\right)^{2}$ by 1 in Eq. (48). Meanwhile, for the matrix elements $\left\langle v_{2} J_{2}\left|\alpha\left(r_{2 N}\right)\right| v_{2}^{\prime} J_{2}^{\prime}\right\rangle^{2}$ appearing in Eq. (51), by considering only the major term with $v_{2}=v_{2}^{\prime}=0$ and ignoring the small angular dependence, one can replace them by a constant $\left\langle 00\left|\alpha\left(r_{2 N}\right)\right| 00\right\rangle$. Then, there is only the partition function of the second $\mathrm{H}_{2} \mathrm{O}$ molecule remaining in the summations over its quantum numbers in Eq. (46) and the result is simply equal to 1 . Thus, except for $\left\langle 00\left|\alpha\left(r_{2 N}\right)\right| 00\right\rangle^{2}$, quantities associated with the second $\mathrm{H}_{2} \mathrm{O}$ molecule are gone. For simplicity we omit the subscript 1 in the following derivations.

Meanwhile, with respect to quantities associated with the first $\mathrm{H}_{2} \mathrm{O}$ molecule, one usually ignores the slight dependence of $\left\langle v J\left|\mu\left(r_{N}\right)\right| v^{\prime} J^{\prime}\right\rangle$ on the rotational quantum numbers and replace them by $\left\langle v 0\left|\mu\left(r_{N}\right)\right| v^{\prime} 0\right\rangle$. In addition, one can replace $C\left(J 1 J^{\prime}, 000\right)^{2}\left\langle v 0\left|\mu\left(r_{N}\right)\right| v^{\prime} 0\right\rangle^{2}$ by the reduced matrix elements $\left|\mu_{i f}\right|^{2}$ of the $\mathrm{H}_{2} \mathrm{O}$ molecule where $i$ stands for $J$ and $\nu$ and $f$ stands for $J^{\prime}$ and $\nu^{\prime}$. We note that there is a symmetry relation

$$
\left(2 J_{i}+1\right)\left|\mu_{i f}\right|^{2}=\left(2 J_{f}+1\right)\left|\mu_{f i}\right|^{2},
$$

which is the same as that between $C\left(J 1 J^{\prime}, 000\right)^{2}\left\langle v 0\left|\mu\left(r_{N}\right)\right| v^{\prime} 0\right\rangle^{2} \quad$ and $\quad C\left(J^{\prime} 1 J, 000\right)^{2}$ $\times\left\langle v^{\prime} 0\left|\mu\left(r_{N}\right)\right| v 0\right\rangle^{2}$. Then, the strength $S_{\left(n_{0}\right)}(T)$ takes a simple form

$$
S_{\left(n_{0}\right)}(T)=2 I(T)\left\langle 00\left|\alpha\left(r_{2 N}\right)\right| 00\right\rangle^{2} P_{i}(T)\left|\mu_{i f}\right|^{2} .
$$

Finally, $\alpha(\omega)$ can be given explicitly as

$$
\begin{aligned}
\alpha(\omega)= & \frac{4 \pi^{2}}{3} \omega\left(1-e^{-\hbar \omega / k_{B} T}\right) \alpha_{F} n_{L}^{2} a_{0}^{5} 2 \alpha_{00}^{2} I(T) \\
& \times \sum_{i f} P_{i}(T)\left|\mu_{i f}\right|^{2} G\left(\omega-\omega_{i f}\right),
\end{aligned}
$$

where $\alpha_{00}\left(=9.922\right.$ a.u.) stands for $\left\langle 00\left|\alpha\left(r_{2 N}\right)\right| 00\right\rangle$. If $G(\omega)$ is available, one can use Eq. (55) to carry out numerical calculations and obtain the CIA spectrum for the $\mathrm{H}_{2} \mathrm{O}-\mathrm{H}_{2} \mathrm{O}$ pair.

\section{The $K_{2}$ line shape model}

There have been many line shape models proposed. ${ }^{25,26}$ Among them, we prefer to consider those that can be well determined. In the present study, we choose the $K_{2}$ line shape model $^{25}$ to represent the normalized line shape function $G(\omega, \eta)$ as

$$
G(\omega, \eta)=G_{0} \cdot \frac{1}{1+e^{-\hbar \omega / k_{B} T}}\left(\frac{\omega}{\eta}\right)^{2} K_{2}\left(\frac{\omega}{\eta}\right),
$$

where $G_{0}$ is a normalization constant with the dimension of $G(\omega, \eta), \eta$ is a parameter with the same dimensions as $\omega$, and $K_{2}$ is a modified Bessel function of the second kind. By exploiting properties of the spectral moments, one can determine the parameter $\eta$ and thus the line shape $G(\omega, \eta)$ itself.

It is well known that the first two moments of the spectral density are defined by ${ }^{13}$

$$
\begin{aligned}
& \Gamma_{0}=\int_{-\infty}^{\infty} F(\omega) d \omega=\int_{0}^{\infty}\left(1+e^{-\hbar \omega / k_{B} T}\right) F(\omega) d \omega, \\
& \Gamma_{1}=\frac{1}{\Delta \omega} \int_{-\infty}^{\infty} F(\omega) \omega d \omega=\frac{1}{\Delta \omega} \int_{0}^{\infty}\left(1-e^{-\hbar \omega / k_{B} T}\right) F(\omega) \omega d \omega .
\end{aligned}
$$

In the above expression, 
TABLE III. Calculated parameters in determining the $\mathrm{K}_{2}$ line shape.

\begin{tabular}{cccccr}
\hline \hline $\begin{array}{c}T \\
(\mathrm{~K})\end{array}$ & $\begin{array}{c}\left\langle R^{-8}\right\rangle \\
\text { (a.u.) }\end{array}$ & $\begin{array}{c}\left\langle R^{-6}\right\rangle \\
\text { (a.u. })\end{array}$ & $\left\langle R^{-8}\right\rangle /\left\langle R^{-6}\right\rangle$ & $\begin{array}{c}C(T) \\
\left(\mathrm{cm}^{-1}\right)\end{array}$ & $\begin{array}{c}\eta \\
\left(\mathrm{cm}^{-1}\right)\end{array}$ \\
\hline 240 & $2.5397 \times 10^{-03}$ & $9.1502 \times 10^{-02}$ & $2.7756 \times 10^{-02}$ & 13.123 & 13.710 \\
270 & $2.2396 \times 10^{-03}$ & $8.1340 \times 10^{-02}$ & $2.7534 \times 10^{-02}$ & 13.018 & 14.474 \\
300 & $2.0322 \times 10^{-03}$ & $7.4270 \times 10^{-02}$ & $2.7362 \times 10^{-02}$ & 12.937 & 15.201 \\
330 & $1.8819 \times 10^{-03}$ & $6.9111 \times 10^{-02}$ & $2.7230 \times 10^{-02}$ & 12.874 & 15.897 \\
\hline \hline
\end{tabular}

$$
\Delta \omega=\sqrt{\frac{3 k_{B} T}{m}} \cdot \frac{1}{\sigma c},
$$

where $m$ is the reduced mass, $c$ is the light speed, and $\sigma$ is the distance where the interaction potential equals zero.

According to the paper by Poll and Hunt, ${ }^{26}$ the moment ratio $\Delta \omega \Gamma_{1}(n) / \Gamma_{0}(n)$ for a general (n) $\left(=\left\{v_{1} v_{2} J_{1} J_{2} v_{1}^{\prime} v_{2}^{\prime} J_{1}^{\prime} J_{2}^{\prime} ; \lambda_{1} \lambda_{2} \Lambda L\right\}\right)$ is given by

$$
\frac{\Delta \omega \Gamma_{1}(n)}{\Gamma_{0}(n)}=\frac{\hbar}{4 \pi c m a_{0}^{2}} \cdot \frac{\left\langle\left[\frac{d B_{n}(R)}{d R}\right]^{2}+\frac{L(L+1)}{R^{2}} B_{n}(R)^{2}\right\rangle}{\left\langle B_{n}(R)^{2}\right\rangle} .
$$

It is easy to show that for the specified $\left(n_{0}\right)$ the ratio becomes

$$
\frac{\Delta \omega \Gamma_{1}}{\Gamma_{0}}=\frac{15 \hbar}{4 \pi c m a_{0}^{2}} \cdot \frac{\left\langle R^{-8}\right\rangle}{\left\langle R^{-6}\right\rangle} .
$$

It is worth mentioning that when calculations of $\left\langle R^{-8}\right\rangle$ and $\left\langle R^{-6}\right\rangle$ are carried out in atomic units, the ratio $\left\langle R^{-8}\right\rangle /\left\langle R^{-6}\right\rangle$ is a dimensionless number.

Meanwhile, by representing the spectral density $F(\omega)$ in terms of the line shape $G(\omega, \eta)$ in Eq. (57), one can derive expressions for the first two moments of the $K_{2}$ line shape function. The results obtained are

$$
\begin{aligned}
& \Gamma_{0}^{\prime}=\frac{3 \pi}{2} G_{0} \eta, \\
& \Delta \omega \Gamma_{1}^{\prime}=G_{0} \eta^{2} D\left(\frac{\eta}{k_{B} T}\right),
\end{aligned}
$$

where $D(\gamma)$, which is common for all temperatures, is defined by

$$
D(\gamma)=\int_{0}^{\infty} \tanh \left(\frac{1}{2} \gamma x\right) x^{3} K_{2}(x) d x .
$$

This can be evaluated numerically. Then, we can express the corresponding ratio as

$$
\frac{\Delta \omega \Gamma_{1}^{\prime}}{\Gamma_{0}^{\prime}}=\frac{2}{3 \pi} \eta D\left(\frac{\eta}{k_{B} T}\right) .
$$

As shown in Eq. (46), except for the line shape function there are no other factors in the expression for $F(\omega)$ depending on $\omega$. This implies that the moments of $F(\omega)$ and the moments of $G(\omega, \eta)$ are closely related. More specifically, the ratios of these moments must be the same. Thus, one obtains an equation from which one is able to find $\eta$,

$$
\eta D\left(\frac{\eta}{k_{B} T}\right)=C(T)
$$

where

$$
C(T)=\frac{45 \hbar}{8 c m a_{0}^{2}} \cdot \frac{\left\langle R^{-8}\right\rangle}{\left\langle R^{-6}\right\rangle} .
$$

We explain how one can completely determine the $K_{2}$ line shape by solving Eq. (64) numerically. First of all, we assume the isotropic potential between two $\mathrm{H}_{2} \mathrm{O}$ molecules takes the $\mathrm{LJ}$ form with the parameters $\sigma=5.008 a_{0}$ and $\varepsilon / k_{B}$ $=380 \mathrm{~K}$. Based on this specified isotropic potential $V_{i s o}(R)$ we can calculate values of $\left\langle R^{-8}\right\rangle,\left\langle R^{-6}\right\rangle$, and $\left\langle R^{-8}\right\rangle /\left\langle R^{-6}\right\rangle$ at four different temperatures $T=240,270,300$, and $330 \mathrm{~K}$. Then, with Eq. (65) we can evaluate $C(T)$ accordingly. Second, by plotting the function $\eta D\left(\eta / k_{B} T\right)$ versus $\eta$ derived for these temperatures and drawing horizontal lines with values of $C(T)$ as their ordinates in the same figure, one can solve Eq. (64) numerically. All these results are listed in Table III. We note that values of $C(T)$ are almost independent of the temperature. It is worth mentioning that as the temperature $T$ varies, the line shape parameter $\eta$ varies almost exactly as $T^{1 / 2}$. For instance, $\eta(T=330 \mathrm{~K}) / \eta(T=240 \mathrm{~K})$ $=1.1711$, while $\sqrt{330 / 240}=1.1726$.

With Eq. (65) one can find the normalization constant $G_{0}$ in terms of the parameter $\eta$ as

$$
G_{0}=\frac{2}{3 \pi \eta} .
$$

Thus, the $K_{2}$ line shape defined by Eq. (56) has been completely determined. We present the normalized line shapes at several temperatures in Fig. 5. In addition, we provide the half-widths derived from the positive wavenumber side and they are $29.869,31.397,32.856$, and $34.255 \mathrm{~cm}^{-1}$. These values vary with $T$ approximately as $T^{1 / 2}$. In fact, we can calculate the ratio of the half-width for $T=330$ and $240 \mathrm{~K}$ which equals to 1.15 and is close to $\sqrt{330 / 240}$ (i.e., 1.17).

\section{Numerical calculations of the CIA spectrum}

After the normalized line shapes are available, we can calculate the CIA spectrum from Eq. (55). First of all, we need to find $I(T)$ which is $\left\langle R^{-6}\right\rangle$ in our notation and are listed in Table III. Second, one has to calculate transition parameters such as the reduced matrix elements and the transition frequencies. In practice, it would be convenient to find these quantities from the HITRAN database. ${ }^{27,28}$ Because only the line parameters associated with positive resonance transi- 


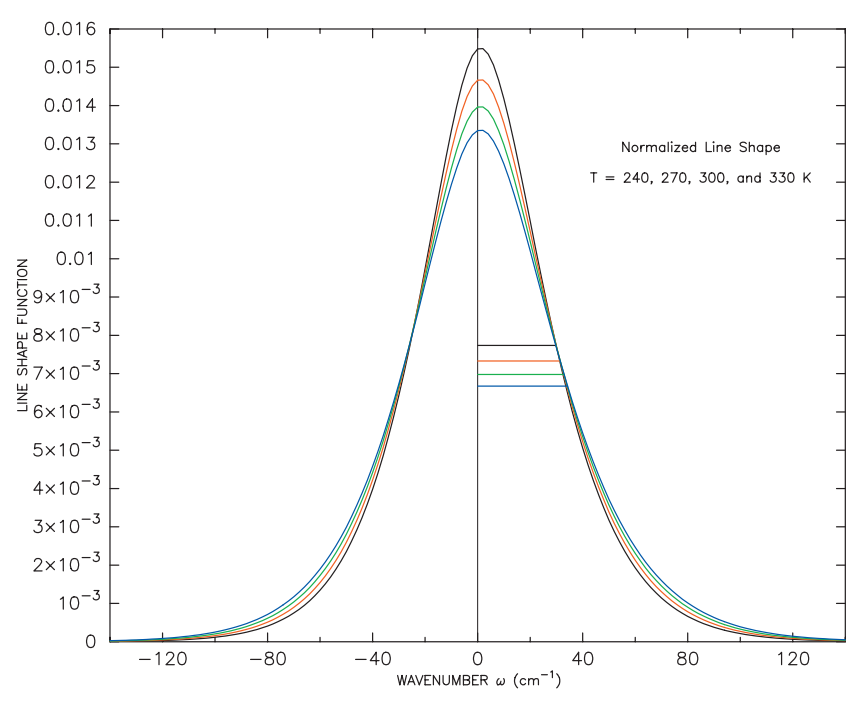

FIG. 5. Calculated normalized $K_{2}$ line shape function $G(\omega, \eta)$ at several temperatures $T=240,270,300$, and $330 \mathrm{~K}$. The line parameters $\eta$ are derived from Eq. (64). These line shapes are normalized. Half-widths derived from the positive wavenumber side of the line shapes are also presented and their values are $29.869,31.397,32.856$, and $34.255 \mathrm{~cm}^{-1}$, respectively. As the temperature $T$ increases, the half-widths increase approximately as $T^{1 / 2}$.

tions are listed in HITRAN, one has to rearrange the summation indices $i$ and $j$ in Eq. (55) accordingly and to rewrite the expression for $\alpha(\omega)$ as

$$
\begin{aligned}
\alpha(\omega)= & \frac{4 \pi^{2}}{3} \omega\left(1-e^{-\hbar \omega / k_{B} T}\right) \alpha_{F} n_{L}^{2} a_{0}^{5} 2 \alpha_{00}^{2} I(T) \\
& \times \sum_{i f ; E_{f}>E_{i}} P_{i}(T)\left|\mu_{i f}\right|^{2}\left\{G\left(\omega-\omega_{i f}\right)\right. \\
& \left.+e^{-\hbar \omega_{i f} / k_{B} T} G\left(\omega+\omega_{i f}\right)\right\} .
\end{aligned}
$$

The line intensity of a transition from an initial state $i$ with lower energy $E_{i}$ to a final state $f$ with higher energy $E_{f}, S_{i f}$ [in units of $\mathrm{cm}^{-1} /\left(\right.$ molecule $\left.\mathrm{cm}^{-2}\right)$ ], listed in HITRAN is given by ${ }^{27,28}$

$$
S_{i f}(T)=I_{a} \frac{4 \pi^{2}}{3 \hbar c} \cdot \omega_{i f}\left(1-e^{-\hbar \omega_{i f} / k_{B} T}\right) \cdot \frac{g_{i} e^{-E_{i} / k_{B} T}}{Q(T)} \cdot R_{i f} \cdot 10^{-36},
$$

where $\omega_{i f}\left(=\left(E_{f}-E_{i}\right) / \hbar>0\right)$ is the resonance frequency, $I_{a}$ $(=0.9973)$ is the natural isotropic abundance, and $g_{i}$ is the total statistical weight of the lower level,

$$
g_{i}=\left(2 J_{i}+1\right) g_{s},
$$

where $g_{s}$ is the nuclear spin degeneracy factor. In the definition of the line intensity given by Eq. (68), $R_{i f}$ are squares of the transition matrix elements given in Debye squared. Because 1 Debye $=10^{-18}$ statcoulomb cm, 1 a.u. $=e a_{0}$ where $e$ is the electric charge and $\left|\mu_{i f}\right|^{2}$ is a dimensionless value measured by $e^{2} a_{0}^{2}$, a comprehensive relationship between $R_{i f}$ and $\left|\mu_{i f}\right|^{2}$ is such that

$$
R_{i f} \cdot 10^{-36}=e^{2} a_{0}^{2}\left|\mu_{i f}\right|^{2} .
$$

With Eqs. (68) and (70), we can find that

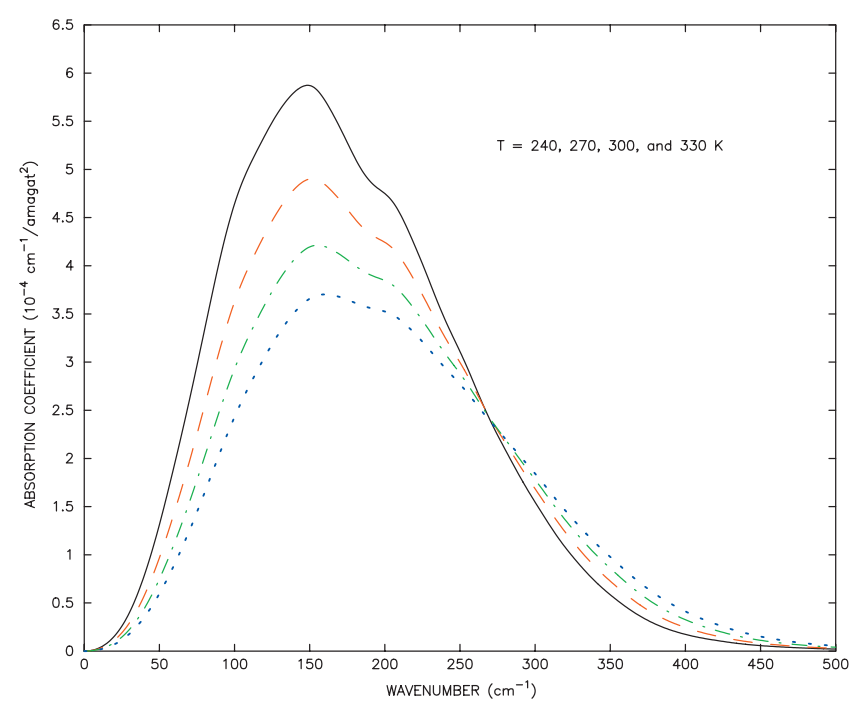

FIG. 6. Calculated CIA spectra in the region from 0 to $500 \mathrm{~cm}^{-1}$ due to the pure rotational band of $\mathrm{H}_{2} \mathrm{O}$ at several temperatures $T=240,270,300$, and $330 \mathrm{~K}$. They are represented by black solid, red dashed, green dot-dashed, and blue dotted lines, respectively.

$$
\frac{4 \pi^{2}}{3} \alpha_{F} a_{0}^{2} P_{i}\left|\mu_{i f}\right|^{2}=\frac{S_{i f}}{\omega_{i f}\left(1-e^{-\hbar \omega_{i f} / k_{B} T}\right)} .
$$

With Eqs. (67) and (71), one obtains an expression for $\alpha(\omega)$ with which one can derive the CIA spectrum based on the $K_{2}$ line shape model,

$$
\begin{aligned}
\alpha(\omega)= & \omega\left(1-e^{-\hbar \omega / k_{B} T}\right) n_{L}^{2} a_{0}^{3} 2 \alpha_{00}^{2} I(T) \\
& \times \sum_{i f ; E_{f}>E_{i}} \frac{S_{i f}}{\omega_{i f}\left(1-e^{-\hbar \omega_{i f} / k_{B} T}\right)}\left[G\left(\omega-\omega_{i f}\right)\right. \\
& \left.+e^{-\hbar \omega_{i f} / k_{B} T} G\left(\omega+\omega_{i f}\right)\right] .
\end{aligned}
$$

First of all, we consider collision-induced transitions resulting from the pure rotational band of $\mathrm{H}_{2} \mathrm{O}$ and calculate the corresponding CIA from Eq. (72) with summations of $S_{\text {if }}$ over this band only. We present calculated results in the region from $0-500 \mathrm{~cm}^{-1}$ at several temperatures $T=240,270$, 300, and $330 \mathrm{~K}$ in Fig. 6. As shown by the figure, the CIA spectra below $275 \mathrm{~cm}^{-1}$ exhibit a negative $T$ dependence, while above they have a positive $T$ dependence. Based on these spectra, one can perform numerical integrations over the wavenumber $\omega$ and obtain values of the integrated absorption coefficients. We present calculated results (in units of $\mathrm{cm}^{-2} /$ amagat ${ }^{2}$ ) in Table IV. Based on these results, it is easy to find their $T$ dependence of the integrated absorption coefficients. It turns out that the integrated absorption coefficients vary as $1 / T^{n}$ with $n \approx 0.94$. It is worth mentioning here that the values of $n$ derived above do vary when different LJ parameters are used in calculations, although the $n$ value would not change dramatically.

TABLE IV. Integrated CIA from 0 to $500 \mathrm{~cm}^{-1}$ at different temperatures.

\begin{tabular}{lcccc}
\hline \hline$T(\mathrm{~K})$ & 240 & 270 & 300 & 330 \\
\hline Integrated CIA & 0.109274 & 0.096752 & 0.088017 & 0.081610 \\
\hline \hline
\end{tabular}




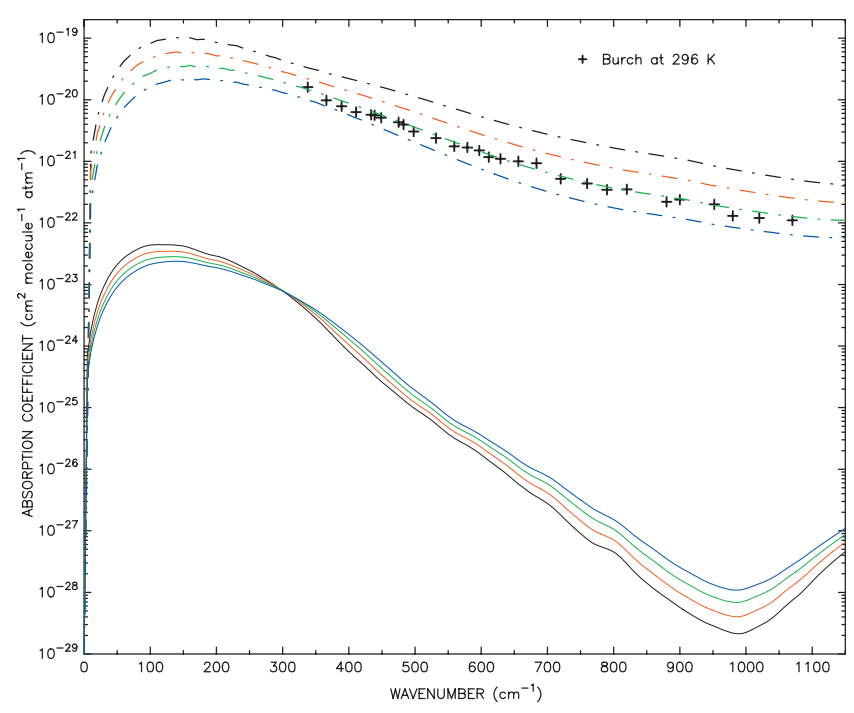

FIG. 7. Calculated CIA spectrum in the region from 0 to $1150 \mathrm{~cm}^{-1}$ at several temperatures $T=240,270,300$, and $330 \mathrm{~K}$. They are represented by black, red, green, and blue lines, respectively. For reference, measurements of the self-continuum absorption coefficients by Burch et al. (Ref. 29) at different temperatures raging from 296 to $353 \mathrm{~K}$ are also presented. In addition, we present predicted values from the MT_CKD model (Ref. 30) at these four temperatures.

Second, we calculate CIA spectra for single transitions of the $\mathrm{H}_{2} \mathrm{O}-\mathrm{H}_{2} \mathrm{O}$ in the region from 0 to $1150 \mathrm{~cm}^{-1}$ at several temperatures $T=240,270,300$, and $330 \mathrm{~K}$ and present results in Fig. 7. In this case, in order to compare the CIA values with other continua provided by measurements and an empirical model, we have made a unit change for $\alpha(\omega)$ from $\mathrm{cm}^{-1} /$ amagat ${ }^{2}$ to $\mathrm{cm}^{2} /$ (molecule atm). We note that the calculated spectra represent contributions to CIA from its major component, i.e., from the (permanent) dipole-induced dipole through the isotropic part of the polarization tensor only. One can identify this origin from two quantities $\left|\mu_{i f}\right|^{2}$ and $\alpha_{00}^{2}$ appearing in Eq. (67) for $\alpha(\omega)$. In addition, it is the presence of $\left|\mu_{i f}\right|^{2}$ that causes a pattern similarity between the CIA and the measured continua shown in Fig. 7. Of course, after accounting for contributions from other CIA components the whole CIA spectra would differ, however, we expect that the pattern would not change dramatically. Concerning the $T$ dependence, as shown in the figure, the CIA spectra within the band center exhibit a negative $T$ dependence while the spectra in the window region have a positive $T$ dependence. However, because the magnitudes of the CIA around the band center are several orders larger than those in the window, the integrated CIA spectra have a negative $T$ dependence. For reference, measurements of the self-continuum absorption by Burch et al. ${ }^{29}$ at different temperatures raging from 296 to $353 \mathrm{~K}$ are also presented. In addition, we present predicted values from the MT_CKD model ${ }^{30}$ at these four temperatures. As shown by the figure, the magnitudes of the CIA spectra are at least two to three orders smaller than the measurements and the empirical model.

\section{E. The $T$ dependence of CIA}

Based on the expression for $\alpha(\omega)$ (in $\mathrm{cm}^{-1} /$ amagat $^{2}$ ) given by Eq. (72), one can conclude that there are several factors that play a role in determining the $T$ dependence of CIA for the $\mathrm{H}_{2} \mathrm{O}-\mathrm{H}_{2} \mathrm{O}$ pair. The factor $\left(1-\exp \left(-\hbar \omega / k_{B} T\right)\right)$ in Eq. (72), except for the low-frequency region and at low temperatures, is always close to 1 . As a result, its role on the $T$ dependence of CIA is very limited. With respect to $I(T)\left(=\left\langle R^{-6}\right\rangle\right)$, we know from Table IV that its value decreases as $T$ increases. More specifically, our numerical results show that as $T$ varies, $I(T)$ varies as $1 / T^{n}$ with $n=0.88$. This means that $I(T)$ causes an uniform negative $T$ dependence of $\alpha(\omega)$ for all frequency regions.

It is worth mentioning here that magnitudes of $I(T)$ and its $T$ dependence characterized by the index $n$ depend on the Lennard-Jones potential parameters $\sigma$ and $\varepsilon$. From numerical tests, the smaller $\sigma$ or the larger $\varepsilon$ is, the larger $I(T)$ is. Meanwhile, with respect to the temperature index $n$, it turns out that $n$ depends sensitively on the Lennard-Jones potential depth $\varepsilon$ and it is almost independent of the separation $\sigma$ at which the potential changes sign. As $\varepsilon$ varies from 300 to $420 \mathrm{~K}$, the corresponding index $n$ varies from 0.64 to 1.01 . We note that $I(T)$ is the dominant factor in determining the $T$ dependence of CIA.

On the other hand, in the summation in Eq. (72) there are two other factors. One is the reduced line intensity defined by Eq. (71) and the other is associated with the normalized line shape function. Generally speaking, line intensities associated with low initial angular quantum numbers $J_{i}$ decrease as $T$ increases and vice versa for those lines with high $J_{i}$. Concerning the normalized line shape, it exhibits different behaviors near the line center and in the wings. More specifically, magnitudes around the line center decrease as $T$ increases and vice versa for the line wings. Of course, the Lennard-Jones parameters $\sigma$ and $\varepsilon$ also affect the line shape because values of $\left\langle R^{-8}\right\rangle$ and $\left\langle R^{-6}\right\rangle$ depend on these two parameters. However, profiles of the line shapes derived from different $\sigma$ and $\varepsilon$ values do not change too much except that some could have wider half-widths than others. As a result, discussions presented here are qualitatively applicable for other choices of the Lennard-Jones parameters. In contrast with the effect from $I(T)$, the effect from the summation of Eq. (72) on the $T$-dependence varies as $\omega$ varies. Because most of strong lines with lower $j_{i}$ values are concentrated near the band center, we expect that the whole effect from these factors would cause a negative $T$ dependence of CIA there. Meanwhile, in the window region we expect the whole effect would yield a positive $T$ dependence which is caused not only by the fact that lines with higher $J_{i}$ values are located there but also because significant contributions to the CIA come from wings of strong lines within the band. These characteristics are well demonstrated by our calculated spectra shown in Figs. 6 and 7.

Finally, as we have explained for the continuum of the far-wing theory in our previous paper ${ }^{1}$ and for the dimer absorption discussed above, the $T$ dependence of $\alpha(\omega)$ depends on the choice of the units. In comparison with the units of $\mathrm{cm}^{-1} /$ amagat $^{2}$ used for $\alpha(\omega)$, the units of $\mathrm{cm}^{2} /($ molecule atm) would introduce an extra factor of $1 / T$. By comparing Figs. 6 and 7, one can find this effect. In general, the negative $T$-dependence within the band center 
becomes stronger and the positive $T$-dependence in the window becomes weaker, but the general pattern remains unchanged.

\section{F. Conclusions on the ClA for the $\mathrm{H}_{2} \mathrm{O}-\mathrm{H}_{2} \mathrm{O}$ pairs}

In the present study, we have used the $K_{2}$ line shape model to carry out numerical calculations because this model is well defined by the two LJ potential parameters. Other line shape models could be used. However, profiles of all these models have similar $T$ dependent patterns: as the temperature increases, their heights become lower and their widths become wider. This implies that basic features of the CIA spectrum and its different $T$ dependences within the band center and at the window are almost independent of the choice of the line shape model. As a result, one can draw general conclusions based on our specific sample calculations.

First of all, in comparisons with other physical mechanisms, contributions to the absorptions in the infrared spectral region from the CIA currently considered here are negligible. We note that in the results presented above, we have neglected the small additional CIA arising from the allowed vibrational matrix elements of the isotropic polarizability between the ground and other vibrational bands, other small contributions arising from the anisotropic polarizability, those due to quadrupolar induction, any short-range induction mechanisms such as the isotropic overlap dipoleinduced dipole, etc. In addition, there are no interference effects occurring between the permanent and transient dipole moments resulting from the multipolar induction mechanism because these two have different spherical harmonic symmetries; the only other type of interference possible is between the long-range part that we included and the short-range part of the dipole-induced dipole that we ignored. Second, with respect to the $T$ dependence of CIA, one can conclude that the CIA has different $T$ dependences for different regions. Within the band center, it has a mild negative $T$ dependence and at the window it exhibits a slightly positive $T$ dependence. However, as a whole CIA (in units of $\mathrm{cm}^{-1} /$ amagat $^{2}$ ) measured by the integrated absorption, it has a mild negative $T$ dependence which can be well described as it varies as $1 / T^{n}$, where $n$ is in the range of $0.6-1.0$.

\section{CONCLUSIONS ON THE THREE PROPOSED MECHANISMS}

From the results shown in paper I (Ref. 1) and the discussion above, we can draw several conclusions. Previously we have studied the continuum from the far wings of allowed lines in the infrared window. Here we have studied the water dimer spectra in the region of $0-600 \mathrm{~cm}^{-1}$ and calculated the CIA spectra from 0 to $1150 \mathrm{~cm}^{-1}$. We note that our studies on these mechanisms do not cover all spectroscopic regions of interested in atmospheric applications. As a result, we will limit our general conclusions accordingly.

First of all, the most important conclusion is that these three mechanisms have completely different $T$ dependences. The dimer spectra exhibit a very strong negative $T$ dependence, the far-wing theory exhibits moderately strong negative one, and the CIA has a weak and mainly negative $T$ dependence. In addition, these three have quite different $T$ dependent patterns, i.e., the strength of its $T$ dependence varies differently as the frequency of interest varies. The farwing theory exhibits the most complex $T$ dependence pattern as shown in Fig. 11 of our previous paper. ${ }^{1}$ On the other hand, the CIA spectra exhibit a systematic $T$ dependence with frequency. Finally, the pattern of the $T$ dependence of the dimer absorptions is rather simpler.

Meanwhile, by comparing their magnitudes we can say a few words on the relative importance for each of these three mechanisms. First of all, we would like to emphasize that when people discuss the continuum, they must consider the local line absorptions as well. This is because the watervapor continua are defined as differences between the raw measured data and calculated local line contributions. However, in order to evaluate the latter, one has to select a cutoff, which is the common practice used, in order to define how the local contributions are calculated. Different choices of the cutoff could yield significantly different results. Besides this technical problem, there are other more profound uncertainties associated with how to depict the line profiles from the center to the cutoff, as well as the accuracies of the spectroscopic parameters used in calculations. Except for window regions between the $\mathrm{H}_{2} \mathrm{O}$ bands, the calculated local line contributions are the largest components of the measured absorptions. This means within the bands, the measured continuum data result from relatively small differences between two large quantities. As a result, these uncertainties, even with relative small percentages, could easily overshadow the measured continua. Given the fact that the continuum from the far wings of allowed lines comes from the same mechanism as the local line absorptions, we prefer to limit comparisons between theoretically calculated continua from the far-wing theory and measurements in the window regions only unless we are able to improve the current accuracy in evaluating the local line absorptions. With respect to the other two mechanisms which are independent of the local line absorptions, one can compare their contributions with measurements for all frequency regions. But, one has also to keep in mind that there are uncertainties of continuum measurements due to the reason mentioned above.

After noting these caveats, we can address the relative importance for each of the three mechanisms. In the $800-1150 \mathrm{~cm}^{-1}$ region, which is the window between the pure rotational band and the $\nu_{2}$ vibrational band, contributions from far wings of allowed $\mathrm{H}_{2} \mathrm{O}$ lines are the dominant source responsible for the continuum. Contributions from the dimer absorption are small or even negligible. ${ }^{8}$ Meanwhile, contributions from the CIA are negligible. In the spectral region between 30 and $500 \mathrm{~cm}^{-1}$, the calculated dimer absorption could account for approximately $20 \%-35 \%$ of the predicted self-continuum from the MT_CKD model at room temperature and account for even more at lower temperatures. After $500 \mathrm{~cm}^{-1}$ they fall off very rapidly as the frequency increases, becoming negligible in the microwindow centered at $944 \mathrm{~cm}^{-1}$. This implies that within the pure rotational band, it is necessary to consider the dimer absorp- 
tions in this region. Meanwhile, in the microwave region the dimer absorptions could make even larger percentage contributions to the self-continuum.

\section{ACKNOWLEDGMENTS}

Two of the authors (Q.M. and R.H.T.) acknowledge financial support from NASA under Grant Nos. NNG06GB23G, NNX09AB62G, and FCCS-547. Q.M. wishes to acknowledge financial support from the Biological and Environmental Research Program (BER), U.S. Department of Energy, Interagency Agreement No. DE-AI0293ER61744. This research used resources of the National Energy Research Scientific Computing Center, which is supported by the Office of Science of the U.S. Department of Energy under Contract No. DE-AC02-05CH11231.

${ }^{1}$ Q. Ma, R. H. Tipping, and C. Leforestier, J. Chem. Phys. 128, 124313 (2008)

${ }^{2}$ Yu. I. Baranov, W. J. Lafferty, G. T. Fraser, Q. Ma, and R. H. Tipping, J. Quant. Spectrosc. Radiat. Transf. 109, 2291 (2008).

${ }^{3}$ W. M. Elsasser, Astrophys. J. 87, 497 (1938).

${ }^{4}$ P. Varanasi, S. Chou, and S. S. Penner, J. Quant. Spectrosc. Radiat. Transf. 8, 1537 (1968).

${ }^{5}$ P. Varanasi, J. Quant. Spectrosc. Radiat. Transf. 40, 169 (1988).

${ }^{6}$ R. E. Roberts, J. A. E. Selby, and L. M. Biberman, Appl. Opt. 15, 2085 (1976)

${ }^{7}$ Y. Scribano, N. Goldman, R. J. Saykally, and C. Leforestier, J. Phys. Chem. A 110, 5411 (2006).

${ }^{8}$ Y. Scribano and C. Leforestier, J. Chem. Phys. 126, 234301 (2007).

${ }^{9}$ J. Boissoles, R. H. Tipping, and C. Boulet, J. Quant. Spectrosc. Radiat. Transf. 51, 615 (1994).

${ }^{10}$ G. Moreau, J. Boissoles, C. Boulet, R. H. Tipping, and Q. Ma, J. Quant. Spectrosc. Radiat. Transf. 64, 87 (2000); G. Moreau, J. Boissoles, R. Le Doucen, C. Boulet, R. H. Tipping, and Q. Ma, ibid. 69, 245 (2001).
${ }^{11}$ J. Boissoles, C. Boulet, R. H. Tipping, A. Brown, and Q. Ma, J. Quant. Spectrosc. Radiat. Transf. 82, 505 (2003).

${ }^{12}$ A. Brown and R. H. Tipping, in Weakly Interacting Pairs: Unconventional Absorbers of Radiation in the Atmosphere, edited by C. CamyPeyret and A. A. Vigasin (Klewer Academic, Dordrecht, 2003), p. 99.

${ }^{13}$ L. Frommhold, Collision-Induced Absorption in Gases (Cambridge University Press, Cambridge, 1993).

${ }^{14}$ P. Demoulin, C. B. Farmer, C. P. Rinsland, and R. Zander, J. Geophys. Res. 96, 13003 (1991).

${ }^{15}$ C. Leforestier, F. Gatti, R. S. Fellers, and R. J. Saykally, J. Chem. Phys. 117, 8710 (2002).

${ }^{16}$ W. Cencek, K. Szalewicz, C. Leforestier, R. van Harrevelt, and A. van der Avoird, Phys. Chem. Chem. Phys. 10, 4716 (2008).

${ }^{17}$ G. T. Evans and V. Vaida, J. Chem. Phys. 113, 6652 (2000).

${ }^{18}$ D. A. McQuarrie, Statistical Mechanics (Harper and Row, New York, 1976).

${ }^{19}$ G. J. Harris, S. Viti, H. Y. Mussa, and J. Tennyson, J. Chem. Phys. 109, 7197 (1998)

${ }^{20}$ R. N. Zare, Angular Momentum (Wiley, New York, 1988).

${ }^{21}$ P. Dore, M. Moraldi, J. D. Poll, and G. Birnbaum, Mol. Phys. 66, 355 (1989).

${ }^{22}$ W. A. Steele, Mol. Phys. 39, 1411 (1980).

${ }^{23}$ M. S. Miller, D. A. McQuarrie, G. Birnbaum, and J. D. Poll, J. Chem. Phys. 57, 618 (1972)

${ }^{24}$ J. D. Poll and J. L. Hunt, Can. J. Phys. 54, 461 (1976).

${ }^{25}$ J. L. Hunt and J. D. Poll, Can. J. Phys. 56, 950 (1978).

${ }^{26}$ J. D. Poll and J. L. Hunt, Can. J. Phys. 59, 1448 (1981).

${ }^{27}$ R. R. Gamache and L. S. Rothman, J. Quant. Spectrosc. Radiat. Transf. 48, 519 (1992).

${ }^{28}$ M. Simeckova, D. Jacquemart, L. S. Rothman, R. R. Gamache, and A. Goldman, J. Quant. Spectrosc. Radiat. Transf. 98, 130 (2006).

${ }^{29}$ D. E. Burch and D. A. Gryvnak, Hanscom AFB Report No. AFGL-TR79-0054, 1979; D. E. Burch, Hanscom AFB Report No. AFGL-TR-810300, 1982; D. E. Burch and R. L. Alt, Hanscom AFB Report AFGLTR-84-0128, 1984.

${ }^{30}$ S. A. Clough, M. W. Shephard, E. J. Mlawer, J. S. Delamere, M. J. Iacono, K. Cady-Pereira, S. Boukabara, and P. D. Brown, J. Quant. Spectrosc. Radiat. Transf. 91, 233 (2005). 